\title{
LA DIFFRAZIONE NEI RILIEVI SISMICI A RIFLESSIONE
}

\author{
Camillo Contini
}

1. Premesse. -- I principi dell'ottica greometrica permettono di rappresentare $\mathrm{i}$ fenomeni di trasmissione delle onde con trande cliatrezza e semplicita ma con approssimazione non sempre sufficiente per le esigenze pratiche. Questa approssimazione si può ritenere inversamente proporzionale alla radice quadrata della lunghezza delle onde considerate, quindi, mentre in genere risulta elevata per i fenomeni della luce, con lunghezze d'onda intorno al mezzo micron, risulta assolutamente grossolana quando si studiano i suoni, con lunghezze di onda comprese fra i 10 cm e i $15 \mathrm{~m}$.

Nella sismica a riflessione si hanno delle lunghezze d'onda ancora maqriori di quelle dei suoni, comprese fra i 10 e i 500 metri, quindi dobliamo allenderci un'approssimazione ancora pegrare.

In effetto l'ottica greometrica permette la determinazione rigorosa delle superfici riflettenti, che particolamente interessa la prospezione sismica, quando tali superfici sono continue, ossia quando wli strati costituenti il soltosuolo presentano delle caratteristiche elastiche e di densità costanti o lentamente variabili nello spazio e sono piegati secondo curve non troppo rapide, senza rotture o discontinuità. In pratica queste condizioni favorevoli raramente risultano verificate, specialmente in corrispondenza alle cosidette "Strutture" che interessano le ricerche petrolifere, teneralmente intersecate da faglie e complicate da erosioni e da discordanze. Insorgono allora dei fenomeni, nella trasmissione e nella riflessione delle onde, molto complessi i quali, se venæono analizzati in hase ai principî dell'otlica geometrica, conducono a delle ricostruzioni delle superfici riflettenti arlitrarie, se non completamente errate.

Come si sa. l'ollica geometrica ammette la trasmissione reltilinea e quindi l'esistenza di zone d'ombra, ossia di zone, coperte da schermi ove le onde risultano assolutamente assenti. Questo fatto è quotidianamente smentito dalla proparazione dei suoni i quali "A Aqgirano " grli ostacoli e dietro di questi, invece di avere il silenzio assoluto. come vorreblue l'ottica greometrica, tutt'al più si ha un indelolimento dei suoni stessi. 
Fenomeni simili si hanno nella sismica a riflessione quando le superfici riflettenti si interrompono bruscamente oppure si interpongono delle masse assorbenti l'energia sismica che funzionano da schermi: oltre le interruzioni delle superfici o i hordi degli schermi dovremmo avere assenza di onde riflesse; invece queste sono presenti e, per di più, con ampiezze tali che possono dare luogo a delle riflessioni all'apparenza ollime sui sismogrammi di registrazione.

Tulti i fenomeni della propagazione dell'energia ragngiante $i$ quali si manifestano con legroi diverse da quelle classiche dellotlica freometrica si riuniscono sotto il nome di "Fenomeni di diffrazione " $0_{4} . \mathrm{em}$ plicemente, di "Diflrazione ".

Noi analizzeremo quindi l'influenza della diffrazione nei rilievi sismici a riflessione in uso nella prospezione mineraria e in particolare nei rilievi delle discontinuití di cui sopra abbiamo detlo, per le quaii l'otlica greometrica riesce insulficiente.

2. Considerazioni sui principî di Huyghens-Fresnel. - Supponiamo, per agevolare le nostre considerazioni, che il terreno ove si trasmettono le onde sismiche sia perfettamente omogreneo ed isotropo. In tale caso, evidentemente, le superfici d'onda sono sferiche col centro nell'origrine dei sismi.

Una perturbazione sinusoidale provocala da uno scoppio $\dot{c}$ rap)presentabile sulle superfici d'onda di ragrio e mediante la formola

$$
E_{\mathrm{o}}=\frac{A}{\varrho} \cos 2 \pi\left(\frac{t}{T}-\frac{\varrho}{\lambda}\right)
$$

ove $t$ sia il tempo misurato dall'istante dello scoppio, i. la lunghezza dell'onda di perturbazione, $T$ il periodo (ossia il tempo di una oscillazione completa) ed $A$ l'ampiezza alla distanza unitaria dallorigine dei sismi, che indicheremo con $S$.

Per i principî di Huygrhens-Fresnel (1) abbiamo che:

1) ogni particella di una superficie d'onda $Q$ è sorğente di nuove onde elementari fra loro coerenti;

2) la superficie a'onda $Q^{\prime}$ successiva alla $Q$ è costituita dall'inviluppo delle onde elementari emesse dai vari punti di $Q$;

3) nei punti ove le diverse onde elementari si sovrappongrono le azioni si compongono secondo la regola dei moti armonici.

L'applicazione più semplice e più conosciuta di tali principî̀ è quella di calcolare le ampiezze delle perturbazioni in un punto $M$ 
qualsiasi dello spazio come somma degli effetti delle singole "Zonc di Fresnel " di una data superficie d'onda $($.

Congiungrimo $M$ colla sorgente $S$ dei sismi; sia $R_{0}$ l'intersezione con $Q$ e sia $\varrho=S R_{0}$ ed $r_{0}=M R_{0}$. Quindi intersechiamo la superficie d'onda Q con delle superfici sferiche aventi i centri in $M$ con raggi $r_{1}=r_{0}+1 / 2 \hat{\lambda}, r_{2}=r_{0}+2 \frac{1}{2} \lambda, \ldots . . r_{14}=r_{0}+n 1 / 2 \hat{i}$. Le superfici anulari individuate su $\Omega$, come si sa, sono le cosidette "Zone di Fresnel ). Vediamo l'influenza di ciascuna di queste zone su $M$.

Suddividiamo una zona qualunque, per esempio la $i$-esima, in zone infinitesime di larghezza udı, essendo $2 *$ langolo sotto cui sono

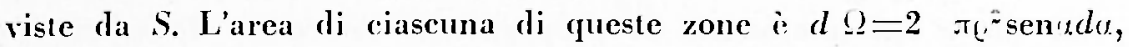
e l'influenza su $M$ è data dalla

$$
d E=\frac{C A}{\varrho r_{\mathrm{i}}} \cos 2 \pi\left(\frac{t}{T}-\frac{\varrho+r_{i}}{i}\right) d \Omega
$$

ove $C$ ì un fattore di proporzionalita. Esprimendo $d \Omega$ in funzione di $d r$ e integrando per tutto l'intervallo fra $r_{\mathrm{i}-1}$ ed $r_{\mathrm{i}}$, posto $C=$ costante, otteniamo per l'intera zona $i-$ esima

$$
L_{\mathrm{i}}=\frac{C_{\mathrm{i}} \lambda A}{\varrho+r_{\mathrm{o}}}\left[\operatorname{sen} 2 \pi\left(\frac{t}{T}-\frac{\varrho+r_{\mathrm{i}-1}}{\lambda}\right)-\operatorname{sen} 2 \pi\left(\frac{t}{T}-\frac{\varrho+r_{\mathrm{i}}}{\lambda}\right)\right]
$$

Le influenze delle varie zone, alternativamente di segno contrario, differiscono in valore assoluto soltanto per il diverso valore della costante $C$, il quale diminuisce al crescere di $i$ e diviene nullo per tutti i punti che stanno sul circolo di contatto della superficie d'onda $\Omega$ col cono ad essa tangente avente il vertice in $M$.

Se ammettiamo che i valori delle costanti $C$ varino linearmente con $i$ gli effeiti su $M$ della seconda zona risultano annullati per interferenza da quelli delle meta contigue della prima e della terza, gli efletti della quarta zona risultano anmullati da quelli della costante metà della terza e della meta contigrua della quinta, e cosi di segruito: restano quindi attivi soltanto grli effetti della prima meta della prima zona e una sottile zona lungro il cerchio di tangenza di cui sopra. Ma l'effetto di questultima ì nullo, dato che la costante $C$ relativa è pure nullat abbiamo atora, per la [2], l'influenza totale della Q

$$
E=-C_{1} \frac{\lambda A}{\varrho+r_{0}} \operatorname{sen} 2 \pi\left(\frac{t}{T}-\frac{\varrho+r_{3}}{\lambda}\right) \text {. }
$$


3. Formola di Kirchhoff e culcoli grafici di Cormu. - La stessa perturbazione di $M$ può essere determinata mediante la formola di Kirchhoff $\left({ }^{2}\right)$.

$$
E=\frac{A}{2 \hbar} ; \int_{\varrho}^{\infty} \frac{1+\cos \gamma}{\varrho r} \operatorname{sen} 2 \pi\left(\frac{t}{T}-\frac{\varphi+r}{h}\right) d \Omega,
$$

ove $r$ sia la distanza da $M$ dei punti $R$ della superficie $\Omega$ e $\gamma$ gri an. goli formati dalle congiungenti $M R$ colle $R S$.

In effet to tale formola ̀̀ rigorosamente applicabile soltanto nei casi di "Propagazione libera " delle onde sferiche e quindi non nei casi che a noi interessano di propagazione ostacolata da schermi o da discontinuità delle superfici riflettenti e del mezzo trasmittente. L'approssimazione raggiungibile è sempre però più che sufficiente per le deduzioni che in pratica possono essere utili. Tale approssimazione sarà lanto maggiore quanto più grandi saranno le dimensioni degli schermi e delle zone libere e quanto più piccole saranno le lunghezze delle onde utilizzate.

Per eseguire praticamente i calcoli delle eccitazioni sismiche $E$ nei punti dello spazio, in presenza di schermi o di discontinuità delle superfici riflettenti o del mezzo trasmittente, mediante la formola di Kirchhoff, possiamo operare nel seguente modo:

I) tracciamo la superficie d'onda $\Omega$ con centro $S$ e tangente al contorno degli schermi o delle linee di discontinuita e calcoliamo le eccitazioni relative ad ogni punto della $\Omega$ stessa;

II) considerando ognuno di questi punti come sorgente virtuale di perturbazione avente effetto proporzionale al valore dell'eccitazione e sfasata rispetto a questa di $1 / 2 \pi$; l'eccitazione nel punto $M$ si ottiene per interferenza delle onde elementari provenienti da tutti i punti di $\Omega$ non coperti dagrli schermi.

Una semplificazione assai notevole, nell'applicazione della formola di Kirchhoff, si ha quando gli schermi o le discontinuità sono infinitamente estesi e presentano $\mathrm{i}$ bordi o $\mathrm{i}$ limiti rettilinei e paralleli fra loro.

In questi casi possiamo sostituire alla superficie dionda $\Omega$ il circolo $\omega$ d'intersezione di essa con un piano $\|$ passante per $S M$ e normale ai bordi indefiniti degli schermi o ai limiti delle dicontinuità, e considerare le eccitazioni come provenienti soltanto dai punti di $\omega$ : otterremo delle ampiezze per le onde sismiche, nei vari punti del piano proporzionali a quelle che si otterrebluero considerando le eccitazioni relative alla superficie $\Omega$. 
Valendoci di questa possibilita calcoliamo graficamente le eccitazioni dei punti di || nel seguente modo, imetodo di Cornul.

Sia $\delta$ una quantita piccola rispetto a $i$, per esempio $\delta=\hbar / 10$; quindi, facendo centro in $M$, intersechiamo il circolo (1) coi circoli di raggi

$$
\begin{aligned}
& r_{1}=r_{\mathrm{o}}+\gamma, \\
& r_{2}=r_{\mathrm{o}}+2 \delta, \\
& \cdots \ldots \ldots \\
& r_{\mathrm{n}}=r_{\mathrm{o}}+n \delta ;
\end{aligned}
$$

$n$ pui essere, per esempio, uguale a 20.

Se indichiamo con $2 \psi_{1}, 2 \%, \ldots .2$ \& g gli angoli solto cui sono viste da $S$ le coppie delle intersezioni dei circoli di raggi $r_{1}$, $r, \ldots \ldots r_{\mathrm{m}}$, rispetivamente, le lunghezze dei vari elementi d'arco individuati su (1) risultano di $\psi_{1} e,\left(\psi_{0}-\psi_{1}\right) e,\left(\psi_{3}-\psi_{0}\right) 0, \ldots \ldots$. $\left(q_{n}-f_{n-1}\right)$ e, essendo genericamente per il circolo di raggio $r_{j}$

$$
\cos \varphi_{i}=\frac{\left(\varrho+r_{0}\right)^{2}+\varphi^{2}-r_{i}^{2}}{2 \varrho\left(\varrho+r_{0}\right)}
$$

Le eccitazioni che $i$ punti di un elemento d'arco inviano in $M$ sono pressoché in accordo di fase: se indichiamo con 9 . la fase del primo elemento individuato dal circolo di raggio $r_{1}$, quelle degli elementi successivi saranno

$$
\begin{aligned}
& \vartheta_{1}=\vartheta_{n}+2 \pi \frac{\delta}{\lambda} . \\
& \vartheta_{2}=\vartheta_{0}+2 \pi \frac{2 \delta}{\lambda}, \\
& \ldots \ldots \ldots \ldots \ldots \ldots . . . \ldots .1) \delta \\
& \vartheta_{n-1}=\vartheta_{0}+2 \pi \frac{(n-1)}{\lambda} .
\end{aligned}
$$

L'eccitazione in $M$ di un elemento d'arco qualunque di $\omega$, individuato dai circoli di raggi $r_{\mathrm{i}}$ ed $r_{\mathrm{i}+1}$, per la formola di Kirchhoff, è con buona approssimazione proporzionale a

$$
\varepsilon_{\mathrm{i}}=\frac{A}{2 \iota_{i}}\left(\varphi_{\mathrm{i}+1}-r_{\mathrm{i}}\right)\left[1+\frac{\left(\varrho+r_{\mathrm{o}}\right)^{2}-\left(\varrho^{2}-r_{\mathrm{i}}^{*}\right.}{2 \varrho r_{\mathrm{i}}}\right] .
$$

Componendo le eccitazioni relative ai vari elementi d'arco colle fasi relative secondo le note regole del parallelogramma, otteniamo una poligonale coi lati continuamente decrescenti con angoli uguali 
a 2 r)/, la quale si avolge gradualmente intomo a due centri $C_{1}$ e $C_{2}$.

Vella fig. 1 riportiamo la poligonale da noi ottenuta ponendo $\varrho=r_{\mathrm{v}}=2500 \mathrm{~m}, \lambda=100 \mathrm{~m}$ e $\delta=10 \mathrm{~m}$.

Le lunghezze dei lati e le grandezze degli angoli della poligonale sono direllamente proporzionali al valore fissato per $\delta$ : riducendo questo valore otteniamo delle poligonali con spigoli sempre meno appariscenti finché al limite, per o che tende a zero, ahhiamo una curva conimua: la cosidetta “Spirale di Comm ». Questa gode delle seguenti proprielà :

a) ogni suo punto $R_{\text {s }}$ corrisponde a un punto $R^{\prime}$ determinato sulla traccia (o) della superficie d'onda $\Omega$;

b) presenta un flesso nel punto $R_{\text {so }}$ corrispondente al punto $R_{0}$ d'intersezione della $S M$ colla superficie d'onda $Q$;

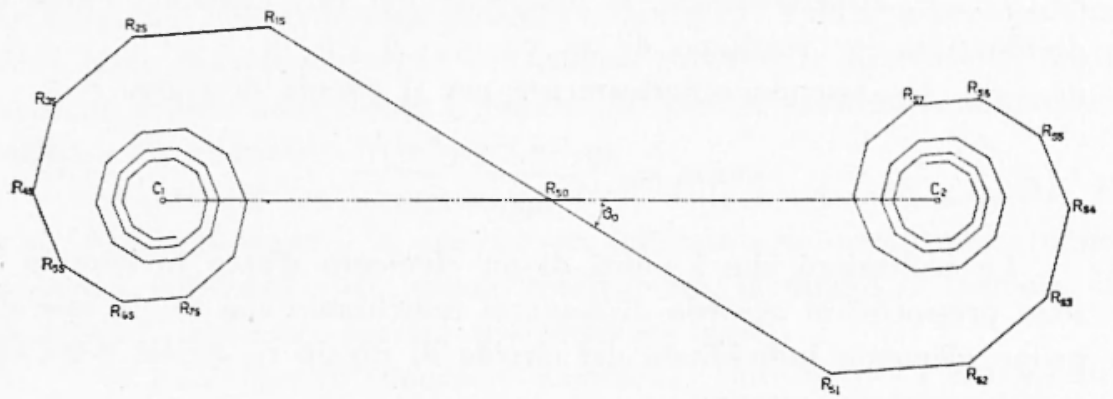

Fig. 1

c) la lunghezza della congiungente i due centri $C_{1}$ e $C_{2}$ (passante per $R_{\text {so }}$ ) rappresenta, secondo un certo coefficiente $K$ di proporzionalita, l'eccitazione in $M$ provocata dall'intera superficie $Q^{6}$ ossia l'eccitazione tolale provocata dallo scoppio di $S$, e, ugualmente, ogni segmento congiungente due suoi punti ̀̀ proporzionale, secondo $K$, all'eccitazione provocata in $M$ dall'elemento d'arco corrispondente di (1);

d) le tangenti in due punti qualunque formano fra loro angoli uguali alle differenze di fase delle eccitazioni inviate in $\boldsymbol{M}$ dai punti corrispondenti di $(1)$; la fase dell'eccitazione totale è data dalla direzione della congiungente $C_{1} C_{\text {: }}$.

Per la pratica, in generale risultano sufficientemente approssimate le spirali a poligonale, simili a quella della fig. 1, che per comodita chiameremo ancora con "Spirali di Cornu ), quando il rapporto $\delta / \lambda$ è scelto abbastanza piccolo, per esempio minore di $1 / 10$.

Nelle considerazioni delle pagine clie seguono noi useremo le 
"Eccitazioni percentuali », che chiameremo ancora con "Ampiezze percentuali », e indicheremo con $h$, le quali si ottengono facendo i rapporti fra i segmenti congiungenti i punti $R_{s 1}$ ed $R_{\mathrm{s} 2}$ di cui $c$ ) e le distanze $C_{1} C_{:}$delle spirali relative e moltiplicando per 100 ; tali rapporli sono indipendenti da $K$.

1. Distribuzione dell'energia delle onde riflesse. - Cerchiamo ora di applicare i principî della diffrazione ai casi della sismica a riflessione e prima di tutto di vedere quale influenza possono avere le eteroxeneiti e le discontinuiti del terreno sulle ampiezze delle onde riflesse alla superficie.

Le eterogeneiti che si presentano più frequentemente in pratica, specialmente nella prospezione petrolifera, sono le inclusioni di forma lenticolare di rocce con caratteristiche clastiche più o meno differenziate rispetto a quelle delle rocce ambiente, originate dalle irregolari variazioni del regime di sedimentazione e particolarmente sviluppate negli strati ad elementi grossolani, conglomerati, ghiaie, sabhie, ecc.

Una lente perfettamente opaca, ossia impermeabile per le onde sismiche, ha l'effello, secondo i principî̀ dell'ottica geometrica, di proietlare un' (Ombra” alla superficie del suolo ove si svolgono le registrazioni dei sismogrammi e quindi di annullare repentinamente le ampiezze delle onde in arrivo. In realta, come vedremo subito, cio non si verifica.

Indichiamo con $d$ il diametro della lente opaca, supposta circolare: la distanza dalla sorgente dei sismi $S$ sia o e quella dai sismograft di registrazione $M$ sia $r_{0}$. Che si tratti di onde dirette oppure di onde riflesse non ha importanza agli effetti delle nostre considerazioni: $\mathbf{i}$ due casi si identificano sostituendo nel secondo caso l'immagine $S^{\prime}$ della sorgente $S$ rispetto alla superficie riflettente. Naturalmente le ampiezze saranno diverse: quelle delle onde riflesse in media dell'ordine del $2 \div 3$ per cento delle ampiezze delle onde dirette.

Se $M$ è sull'asse del cono dell'ombra geometrica della lente, facendo centro in $M$ stesso intersechiamo la parte lasciata libera della superficie d'onda $Q$ che si appoggia all'orlo della lente con i circoli di raggio $r_{1}^{\prime}=r_{0}{ }^{\prime} 1 / 2 \lambda, r_{2}^{\prime}=r_{0}{ }^{\prime}+1 / 22 \lambda, \ldots r_{\mathrm{n}}{ }^{\prime}=r_{0}{ }^{\prime}+1 / 2 m \lambda$, ore $r_{0}^{\prime}$ sia il raggio del circolo tangente all'orlo della lente, determiniamo le zone di Fresnel che risultano attive su $M$. Ripetendo il ragionamento fatto al n. 2 troviamo che in effetto le azioni delle zone si annullano vicendevolmente eccettuata quella della mezza zona a contalto colla lente. 
Se il diametro d della lente $\grave{e}$ piccolo in confronto ad $r_{0}$ la costante $C_{1}$ della $[3]$ non sarà sensibilmente diversa da quella rclativa alla zona adiacente alla lente e l'eccitazione in $M$ risulterà pressoché uguale a quella che si avrebbe se la lente non esistesse.

Aumentando il diametro $d$ l'eccitazione di $M$ diminuirà sempre più: si dimostra però che la velocita della diminuzione ì tanto magrgiore quanto minore i la lunghezza $\lambda$ delle onde considerate.

Ugualmente si dimostra che se ci spostiamo dall'asse dell'ombra geometrica troviamo una graduale diminuzione dell'eccitazione fino al limite dell'ombra stessa: poi un rapido aumento, un'altra diminuzione, e cosi via, similmente a quanto aviene in oltica col fenomeno delle frange di diffrazione. Quando le dimensioni della lente sono abhastanza grandi si hanno i fenomeni delle frange anche all'interno dell'ombra geometrica.

In generale le lenti non sono completamente opache, come abhiamo supposto. L'energia che lasciano passare peró risente l'effetto convergente o divergente delle superfici più o meno regolarmente convesse delle lenti stesse: convergenti quando la velocità di trasmissione del terreno entro le lenti $v^{\prime}$ è maggiore di quella $v$ del terreno fuori, divergente nel caso contrario $v>v^{\prime}$.

Se indichiamo con $=$ i raggi di curvatura medi delle due superfici limite delle lenti e con $f$ la distanza focale $\grave{e}$, evidentemente,

$$
f=\frac{\dot{\varepsilon}}{2} \frac{v}{v^{\prime}-v} .
$$

Quando $f$ fosse molto piccolo in confronto a $\varrho$ e ad $r_{\text {a, }}$ per esempio minore del terzo, le lenti anche se convergenti disperderebhero l'energia come se fossero divergenti. In ogni caso però, sia che le lenti concentrino oppure disperdano l'energia, alla superficie non vi saranno delle variazioni repentine o molto grandi dell'energia stessa in quanto la diffrazione, cosi come colma tutte le zone d'ombra, impedisce le forti concentrazioni dell'energia. E facile dimostrare infatti che appena si dovessero verificare delle concentrazioni (o depauperazioni) d'energia le particelle delle superfici d'onda relative irradierebbero nella direzione delle zone depauperate (o di forte concentrazione) più (o meno) energia di quanta ne riceverehbero dai punti delle stesse zone per cui immediatamente avreble inizio la fase della dispersione (o della concentrazione) e quindi del livellamento dell'energia. 
Quando le lenti fossero di origine vulcanica, dovute a intrusioni filoniane o ad effusioni laviche, oppure di origrine tellonica, dovute a pieglie rotte per stiramento o per farlic o isolate dall'erosione le differenze delle velocità di trasmissione rispetto a quella della roccia ambiente in generale sarebbero forti e le curvature assai notevoli e quindi si avreble un effetto disperdente fortissimo.

Se le lenti sono molto numerose l'effetto è di un sensibile livellamento dell'energia alla superficie del suolo e di una diminuzione renerale dell'ampiezza, cosi come avviene per la luce filtrata attraverso un vetro smerigliato.

Elletti simili a quelli delle lenti si hanno per le stratificazioni a incastri ripetuti e incrociala caratteristiche dei depositi alluvionali e della sedimentazione deltizia, in quest'ultimo caso con grandezzo di solito molto sensibili date le notevoli differenziazioni delle caratteristiche litologiche.

Ugualmente avviene per i depositi eolici, di löss, sabbiosi o piroclastici, per i hanchi depositati dalle correnti marine in prossimita delle foci dei fumi con grande apporto di materiale, per gri ammassi calcarei o silicei di origine organica, per gli ammassi conglomeratici, per le eterogeneità dovute ai diversi gradi di cementazione, ecc.

Le dicontinuità degli strati che più frequentemente occorrono sono le faglie; ma anche altre specie possono avere molta importanza, quali quelle dovute alle discordanze dei terreni che si riscontrano nella zona apicale di molte strutture, alle irregolarità topografiche delle superfici di depositazione dei sedimenti, in particolare vecchie ripe di spiaggria, alle inclusioni di rocce eterogenee, ecc.

Ora consideriamo il caso semplice di una superficie riflettente delle onde sismiche $\pi_{1}$ piana e orizzontale interrotta, per una qualsivorlia ragrione, secondo una linea retta $p_{1}$ e determiniamo le ampiezze delle onde riflesse alla superficie del suolo.

Sia $k$ la profondita di $\pi_{1}$ misurata rispetto alla superficie del suolo $s$ e supponiamo che i sismografi di registrazione $M$ siano posti molto vicini alla sorgente $S$ dei sismi, tanto da poter essere considerati, arli effetti pratici, come coincidenti colla $S$ stessa.

Scoppiando in punti molto lontani dalla retta $p_{0}$, proiezione verticale di $p_{1}$, su $s$, dalla parte opposta a quella ove si trova il semipiano riflettente $\pi_{1}$ evidentemente si ottengono delle onde riflesse di ampiezza nulla, scoppiando viceversa molto lontano dalla parte di $\pi_{1}$ si ottengono delle ampiezze uguali a 100 , rappresentate dalle congiun- 
genti $C_{1} C_{\text {: }}$ delle spirali di Cornn, e scoppiando sulla $p_{\%}$, poiché ogni punto $P_{0}$ di questa intersezione coi piani perpendicolari corrispondono ai punti $R_{s o}$ delle spirali relative agli stessi piani !, si ottengono delle ampiezze percentuali uguali a 50 , naturalmente nell'ipolesi di operare sempre nelle stesse condizioni. Per gli altri punti di $s$ le ampiezze percentuali si determinano nel seguente modo.

(1) Costruiamo la spirale di Cormu corrispondente alla lunghezza d'onda $\lambda$ e alla profondita $k$ che ci interessano ponendo $\varrho=r_{0}=k$.

(i) Proiettiamo da $P_{0}$ i punti $R^{\prime}$ del circolo (1) di raggio $\imath=r_{0}=k$, corrispondenti ai vertici della spirale costruita, sulla superficie riflettente $\pi_{1}$ e sul prolungamento della stessa oltre la retta $p_{1}$ : i punti $R_{11}, R_{12}, R_{13}, \ldots \ldots R_{11}, R_{2.2}, R_{23}, \ldots$. che si ottengono sono pure corrispondenti ai rertici successivi della spirale di Corm, $\boldsymbol{R}_{1 \mathrm{~s}}, \boldsymbol{R}_{\mathrm{ss}}, \boldsymbol{R}_{3 \mathrm{~s}}, \ldots$ ed $\boldsymbol{R}_{\mathrm{s} 1}, \boldsymbol{R}_{\mathrm{s} s}, \boldsymbol{R}_{\mathrm{s} 3}, \ldots$ dalle parti di $C_{1}$ e di $\boldsymbol{C}_{:}$ rispettivamente; evidentemente la traceia $P$ della rella $p_{1}$ d'intersezione col piano perpendicolare $\|$, cui è relativa la spirale, è corrispondente ad $R_{\text {so. }}$ Posto $\delta_{1}=P R_{11}=P R_{21}, \delta_{2}=P R_{12}=P R_{22}, \delta_{3}=P R_{13}=P R_{23,}$,

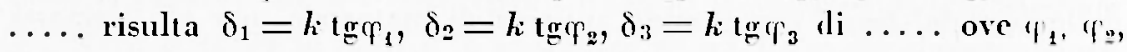
$\varphi_{3}, \ldots$. sono ottenuti mediante la $[5]$.

$\gamma)$ Sulle ordinate tracciate per i punti $P, R_{11}, R_{12}, R_{13:} \ldots \ldots$ $R_{21}, R_{22}, R_{23}, \ldots$ portiamo a partire da $s$ i valori delle distanze dei punti corrispondenti della spirale di Corm dal centro $C$; forrispondente all'infinito dalla parte opposta di $\pi_{1}$ i, moltiplicati per 100 e ridotti del valore della congiungente $C_{1} C_{2:}$.

Nelle figure $2 a$ ), b e e riportiamo le curve ottenute per le profondità $h$ rispettivamente di 1000,2500 e 5000 metri, relative ai valori delle lunghezze d'onda $\lambda$ di 25, 50, 100, 200 e 400 metri.

Caratteristiche notevoli di tali curve sono:

A) presentano, a partire dalla verticale per la linea di discontinuità $p_{1}$, dalla parte di $\pi_{1}$, successivamente i massimi relativi li $h_{1}=117, h_{2}=109, h_{3}=107, h_{4}=105,5, \ldots$ e successivamente i minimi relativi di $h_{1}{ }^{\prime}=88,5, h_{0}{ }^{\prime}=92, h_{3}{ }^{\prime}=94, \ldots \ldots$;

B) si trasformano luna nell'altra variando per le stesse profondità $k$ le ascisse (misurate dalla verticale per $p_{1}$ ) del rapporto delle radici quadrate delle lunghezze d'onda relative e per profondità $k$ diverse variando le lunghezze d'onda del rapporto delle profondità; per esempio, per og̣ni profondità possiamo ottenere la curva relativa a $\lambda=200 \mathrm{~m}$ adottando $\mathrm{i}$ valori delle ordinate della curva relativa a $\lambda=50 \mathrm{~m}$ e dei valori doppi per le ascisse corrispondenti; 


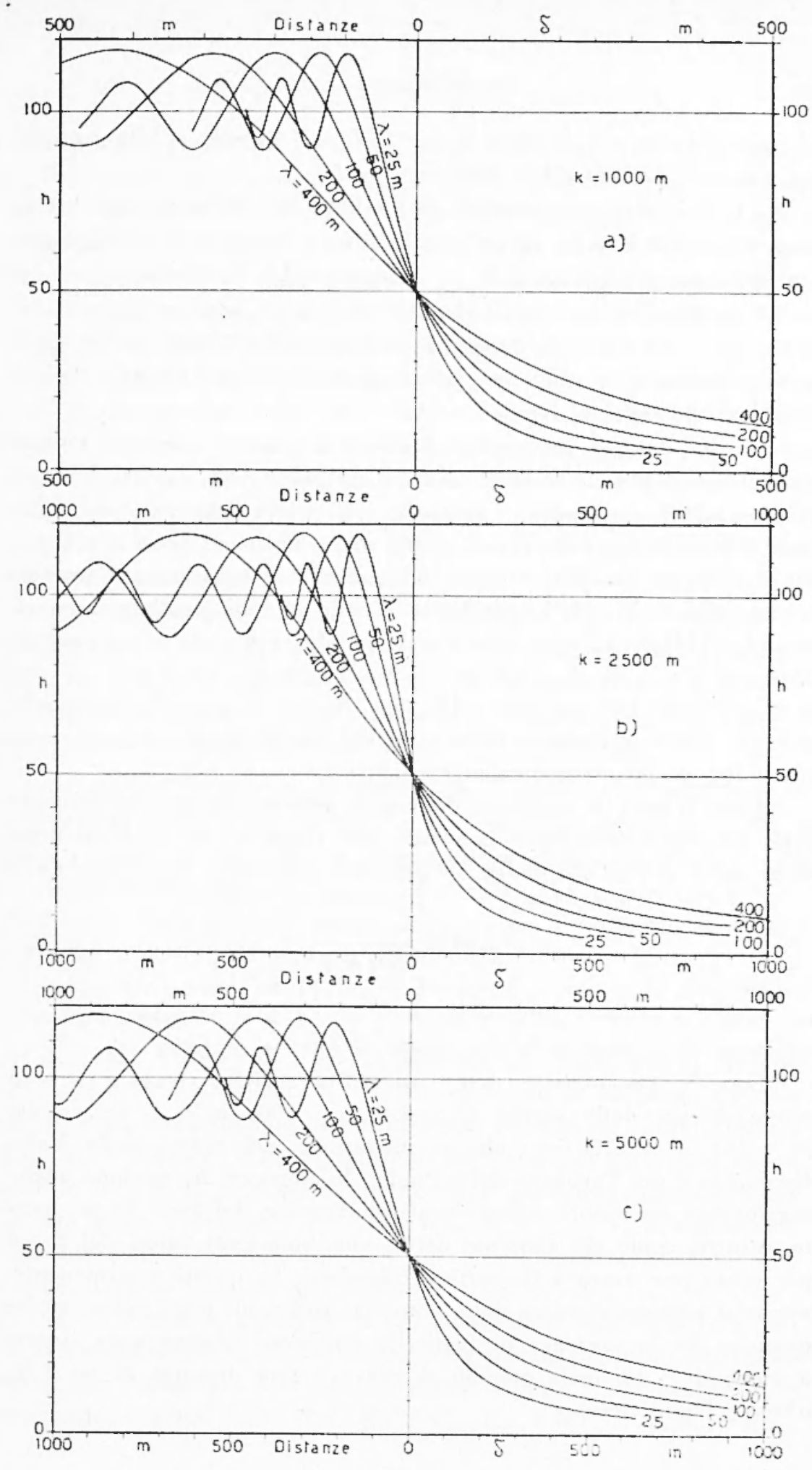

Fig. 2 
la curva relativa a $\lambda=200 \mathrm{~m}$ e $k=2500 \mathrm{~m}$ è sovrapponilbile a quella relativa a $\lambda=100 \mathrm{~m}$ e $h=5000 \mathrm{~m}$;

$C$ ) le distanze orizzontali dalla linea di faglia $p$, del primo massimo relativo delle curve, per le diverse profondita e lunghezze d'onda, sono pressapoco di $\delta_{\mathrm{m}}=-0,625 \sqrt{i k \mathrm{k}}$.

Naturalmente tali caratteristiche risultano verificate nelle curve della fig. 2 entro i limiti dell'approssimazione dei calcoli grafici, piuttosto grossolana, la quale peggiora rapidamente per $i$ valori suecessivi al primo minimo relativo.

Risulta dunque che, contrariamente a quanto vorrebhe l'ottica geometrica, l'approssimarsi di una discontinuità della superficie riflettente si manifesta dapprima con delle oscillazioni delle ampiezze delle onde riflesse e poi, dalla distanza $\delta_{m}$, di cui ablbiamo detto in $C$, con un decremento continuo di entità pressoché inversamente proporzionale alla radice delle lunghezze d'onda e delle profondita considerate: ab),iamo in ogni caso che le ampiezze $h$ sono pressapoco di 0,90 per $\delta=+1 / 2 \quad \delta_{\mathrm{m}}, 0,70$ per $\delta=+\delta_{\mathrm{m}} 4,1 / 2$ per $\delta=0,1 / 3$ per $\delta=-3 \delta_{\mathrm{m}} / 10,1 / 5$ per $\delta=-4 \delta_{\mathrm{m}} / 5,1 / 10$ per $\delta=-8 \delta_{\mathrm{m}} / 5$, essendo indicate con $\delta$ le distanze dalla verticale per la faglia col verso positivo dalla parte ove manca la superficie $\pi_{1}$.

Come è noto, le ampiezze delle onde riflesse, espresse in percento delle ampiezze delle onde incidenti, per rispetto alle caratteristiche delle rocce a contatto lungo le superfici riflettenti, sono date dalla

$$
h_{\mathrm{r}}=100 \frac{\sigma v-\sigma^{\prime} v^{\prime}}{\sigma v+\sigma^{\prime} v^{\prime}}
$$

ove con $\sigma$ e $r^{\prime}$ sono indicate le densità e $v$ e $v^{\prime}$ le velocità di trasmissione delle onde delle due specie di rocee a contatto.

Quando queste roce sono molto differenziate, come per esempio ì il caso delle argille $(\sigma=2,40, v=2000 \mathrm{~m} / \mathrm{s})$ e dei calcari $\left(\sigma^{\prime}=2,70, v^{\prime}=5200 \mathrm{~m} / \mathrm{s}\right)$ che si incontrano nei rilievi della Valle Bradanica e del Tavoliere delle Puglie, le ampiezze $h_{\mathrm{r}}$, possono anche raggiungere dei valori molto elevati, dell'ordine del $20 \div 40$ per cento, mentre, come gia abbiamo detto, sono sufficienti valori del $2 \div 4$ per cento per avere delle ottime riflessioni. In questi casi evidentemente si possono ottenere delle ampiezze sufficienti per i rilievi anche quando, per la presenza di faglie, le ampiezze relative sono ridotte a meno di $1 / 10$, ossia quando si rileva a una distanza di $-2 \mathrm{~lm}$ oltre le faglie stesse. 
Nel campo dei fenomeni di trasmissione della luce ci mettiamo in condizioni simili a quelle che si lanno coll'interruzione della superficie riflettente $\pi_{1}$ immaginando di porre un diaframma con bordo rettilineo innanzi a una sorgente puntiforme: su uno schermo posto dietro il diaframma si manifestano, in vicinanza del limite dell'ombra geometrica e dalla parte della zona illuminata, delle frange alternativamente brillanti e oscure, il cui contrasto si attenua sempre più finché si ha un'illuminazione pressoché uniforme, mentre nell'interno dell'ombra geometrica la luce diminuisce gradualmente senza dar luogo a massimi e minimi. L'analogia con quanto avviene per le onde sismiche riflesse dal semipiano $\pi_{1} \grave{c}$ evidente. La dipendenza dell'intensiti dei fenomeni della diffrazione dalle lunghezze dellc onde è dimostrata dal fatto che se la sorgente è di luce bianca anziché monocromatica le frange si manifestano colorate.

La configurazione strutturale a faglia si può schematizzare in quella che risulta dalla suddivisione di un piano in due semipiani $\pi_{1}$ e $\pi_{y}$ e dallo spostamento relativo di questi in senso verticale e orizzontale. Ognuno dei due semipiani si comporterà evidentemente nello stesso modo che abbiamo visto.

In pratica raramente le rolture degli strati sono semplici: in gencrale le faglie sono multiple e si presentano secondo sistemi, i quali rendono le zone ove si estendono pressoché opache, incapaci di rifleltere quantità apprezzabili d'energia. Per comprendere bene che cosa avvenga in simili casi studiamo prima l'effetto che si ottiene supponendo che un dato piano riflettente $\tau$ sia reso assorbente (opaco) lungo una striscia delimitata dalle rette $p_{1}$ e $p_{2}$ alla distanza $\sigma$ l'una dall'altra.

Il piano riflettente $\pi$ sia orizzontale e la profondità, rispetto alla superficie $s$, sia ancora $k$, Quindi, operando in un piano nonnale alle $p_{1}$ e $p$. , immaginiamo di eseguire una costruzione simile a quella della fig. 3. Siano $P_{1}$ e $P_{2}$, equidistanti da $P$, i limiti della zona opaca, ossia le traccie di $p_{1}$ e $p_{2}$. A partire da $P$ portiamo successivamente da una parte $\mathrm{i}$ segmenti $P R_{1}{ }^{\prime}, R_{1}{ }^{\prime} R_{2}{ }^{\prime}, R_{2}{ }^{\prime} R_{3}{ }^{\prime}, \ldots$ e dall'altra i seymenti $P R_{1}^{\prime \prime}, R_{1}^{\prime \prime} R_{2}^{\prime \prime}, R_{2}^{\prime \prime} R_{3}^{\prime \prime}, \ldots \ldots$ di lunghezza costante $1 / 2 \sigma$ e sulle ordinate tracciate per i punti $P, R_{1}{ }^{\prime}, R_{2}^{\prime}, R_{3}{ }^{\prime}, \ldots R_{1}^{\prime \prime}, R_{2}^{\prime \prime}$, $R_{:}{ }^{\prime \prime}, \ldots$ portiamo, a partire da $s$, i valori delle differenze veltoriali fra la congiungente $C_{1} C_{2}$ dei centri della spirale di Cornu corrispondente alla profondita $h$ e alla lunghezza d'onda $\lambda$ considerate e le congiungenti i punti $R_{\mathrm{s} 1}{ }^{\prime}$ e $R_{\mathrm{s} 1}{ }^{\prime \prime}, R_{\mathrm{s} 2}{ }^{\prime}$ e $R_{\mathrm{s} 0}, R_{\mathrm{s} ;}^{\prime}$ e $R_{\mathrm{s} 1}{ }^{\prime}, \ldots \ldots R_{\mathrm{so}}$ e $R_{\mathrm{s} 2}{ }^{\prime \prime}$, 
$R_{\mathrm{s} 1}^{\prime \prime}$ e $R_{\mathrm{s}, 3}{ }^{\prime \prime}, \ldots$ della stessa spirale corrispondenti ai punti $R_{1}{ }^{\prime}$ e $R_{2}^{\prime \prime}$, $R_{2}^{\prime}$ e $P, R_{3}^{\prime}$ e $R_{1}^{\prime}, \ldots \ldots P$ e $R_{2}^{\prime \prime}, R_{1}^{\prime \prime}$ e $R_{3}{ }^{\prime \prime}, \ldots$ rispeltivamente, divisi per il valore della congiungente $C_{1} C_{0}$ e moltiplicati per 100 .

La curva che si ottiene è simmetrica rispetto alla ordinata per $P$. Quando $\sigma$ fosse molto grande in confronto alla profondita $k$ naturalmente si adotteranno per le lunghezze dei segmenti $P R_{1}{ }^{\prime}, R_{1}{ }^{\prime} R_{2}{ }^{\prime}, \ldots \ldots$ $P R_{1}{ }^{\prime \prime}, R_{1}{ }^{\prime \prime} R_{2}{ }^{\prime \prime}, \ldots$ delle frazioni di $\sigma$ stesso minori di $1 / 2$, per esempio $1 / 4$ oppure $1 / 8$.

Nella fig. 3 riportiamo le curve delle ampiezze ottenute per la profondità $k$ di $2500 \mathrm{~m}$, la lunghezza d'onda $\lambda$ di $100 \mathrm{~m}$ e per le

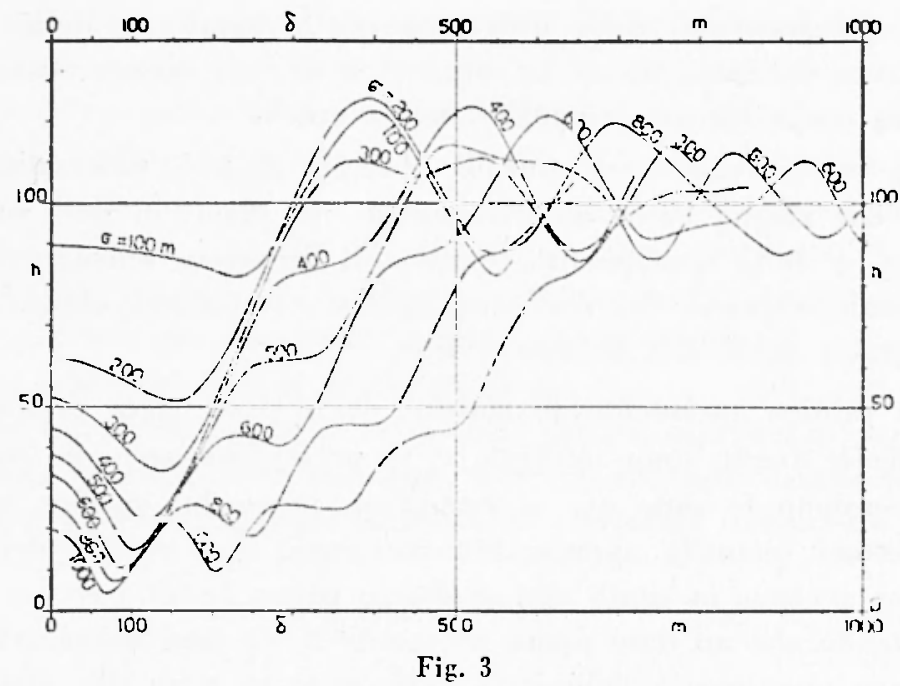

larghezze $\sigma$ delle striscie opache di $100,200,300,400,500,600,800$ e $1000 \mathrm{~m}$.

Vediamo che, al solito, variando le distanze $\delta$ dalla mezzaria delle striscie le ampiezze si avvicinano alla retta $h=100$ passanło attraverso massimi e minimi relativi, corrispondenti alle frange d'interferenza che si osservano, in condizioni analoghe, per $\mathrm{i}$ fenomeni luminosi.

Contrariamente a quanto apparirebbe a prima vista, i valori minimi delle intensità non cadono in corrispondenza alle mezzarie delle striscie, $\delta=0$, ma in corrispondenza a due punti simmetrici rispetto alle mezzarie stesse: le ascisse $\delta_{\mathrm{m}}{ }^{\prime}$ di detti punti sono approssimativamente decrescenti secondo la radice dell'inverso delle larghezze $\sigma$ 
delle striscic e urualmente aviene per i rapporti fra le ampiezze minime e quelle corrispondenti alle mezzarie.

Le curve della fig. 3 sono valide per qualsiasi profondità $k$, per Iunghezze d'onda però tali per cui la radice del prodotto ik risulti costante ed uguale a quella relativa a $t=100 \mathrm{~m}$ e $k=2500 \mathrm{~m}$. Restando invariata la profondità $k$ otteniano le curve per valori diversi dalle lunghezze d'onda i. moltiplicando i valori di o segnali sulle ascisse e quelli di $\sigma$ segnati sulle curve per il rapporto a 100 degli stessi valori delle lunghezze d'ondil.

Date queste proprietà possiamo evidentemente determinare le ampiezze relative a lutle le profondita e lunghezze d'onda che in pratica possono interessare.

Vediamo dagli andamenti delle curve che le riduzioni dell'energria riflessa alla superficie del suolo $s$ in vicinanza $e$ in corrispondenza alle striscie opache, per le larohezze $\sigma$ minori di $800 \div 1000$ metri, sono relativamente scarse, tali da non escludere la possibilità di rilevare delle luone riflessioni, specialmente per le piì hasse frequenze utilizzabili colle più moderne apparecchiature di registrazione, appena le differenziazioni dei terreni risultano discrete.

Praticamente si poseono avere effetti simili a quelli rli un piano riflettente opaco lungo una striscia colla configurazione strutturale che i geologi indicano con "Graben " e quella opposta, di una fetta di strati sopraelevata limitata da faglie, per le onde riflesse dai lembi corrispondenti esterni alle faglie. Se però questi lembi non sono arrli stessi livelli, l'uno in prosecuzione dell'altro, gli effetti risultano molto diversi: sui sismogrammi le riflessioni iniziano piuttosto debolmente colle onde riflesse dal lembo più vicino allorigine d vi sirmi e agli strumenti di registrazione, poi si ha $n$ aumento delle ampiezze per il sopraggiungere delle onde riflesse dall'altro lembo: queste in generale hamo fasi diverse da quelle delle prime onde, quindi, data l'interferenza che ha luogo, si hanno rapide variazioni delle fasi stesse. Vedremo in seguito che in realtà le riflessioni risultano ancor più complicate per il fatto che le ampiezze delle onde non sono costanti nel tempo.

Vediamo ora quali siano invece gli effetti che si hanno dalle striscie delimitate dalle faglie, delle configurazioni di cui sopra abbiamo detto, supposte riftettenti, ossia nell'ipotesi che il piano $\pi$ sia riflettente soltanto lungo la striscia delimitata dalle relte $p_{1}$ e $p_{2}$ alla distanza $\sigma$ l'una dall'altra. 
In questi casi le ampiezze percentuali sono date dai valori, moltiplicati per 100 e divisi per $C_{1} C_{2}$, delle congiungenti i punti $R_{\mathrm{r}}$ '

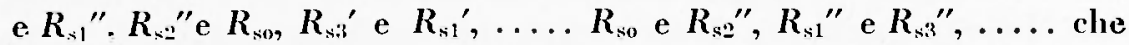
nel caso della striscia opaca si sono sottratti vettorialmente a quelli della congiungente $C_{1} C_{:}$. La curva delle ampiezze è evidentemente

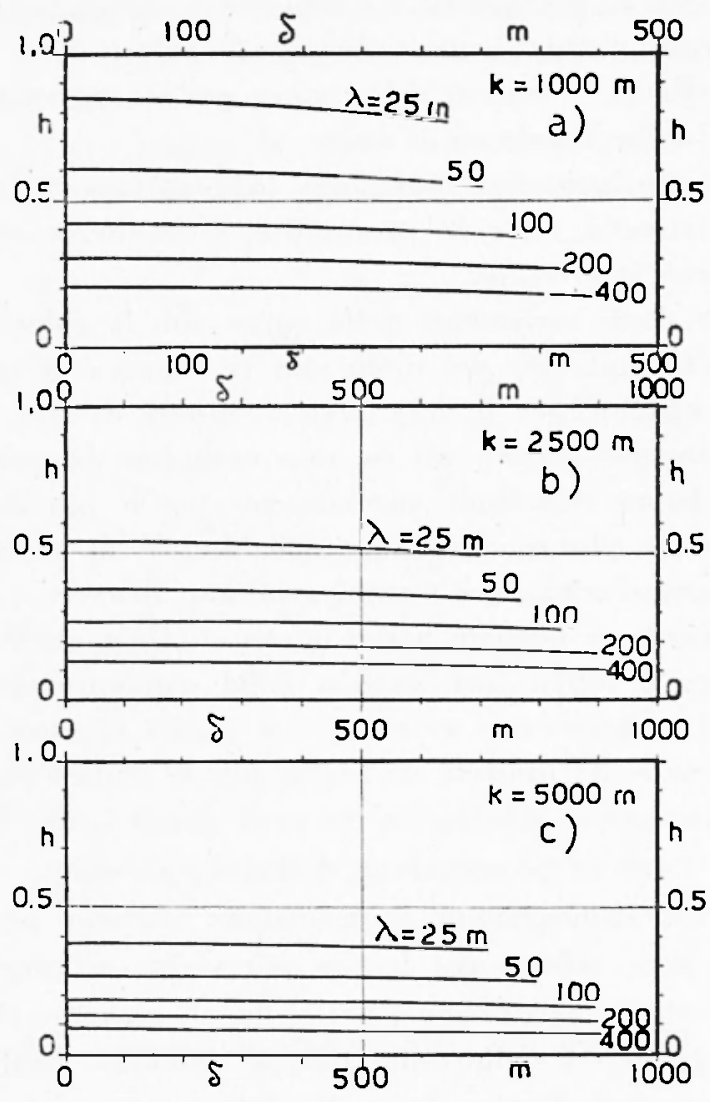

Fig. 1

simmetrica rispetto all'ordinata per $P$, corrispondente alla mezzaria della striscia riflettente.

Se la larghezza $\sigma$ della striscia è molı grande in confronto alla profondità le ampiezze delle onde riflesse in superficie in prossinità delle verticali per i bordi e oltre nella zona dellombra geometrica risultano pressapoco uguali a quelle che si hanno in condizioni analoghe dalle curve della fig. 2. Per il caso contrario, di una larghezza $\sigma$ piccolisima, uguale a 1 metro, otteniamo le curve della fig. 4: per 
tali curve si sono considerate le lunghezze d'onda di 25, 50, 100, 200 e 400 metri, per le profondita solite di $1000 \mathrm{~m}$, curve a), di $2500 \mathrm{~m}$. curve $b$ ), e di $5000 \mathrm{~m}$, curve $c$ ). Risulta, come è facile verificare, che le ampiezze sono inversamente proporzionali a $\sqrt{\hat{h}} \cdot \mathbf{k}$.

Per le larghezze della striscia piccole, per esempio minori di $10^{-1} V \bar{\lambda} \cdot \hat{k}$, si possono determinare approssimativamente gli effelti alle diverse distanze moltiplicando $i$ valori di $h$ dati dalle curve della figr. + per $\sigma$. Per i valori maggiori delle larghezze gli effelti saranno

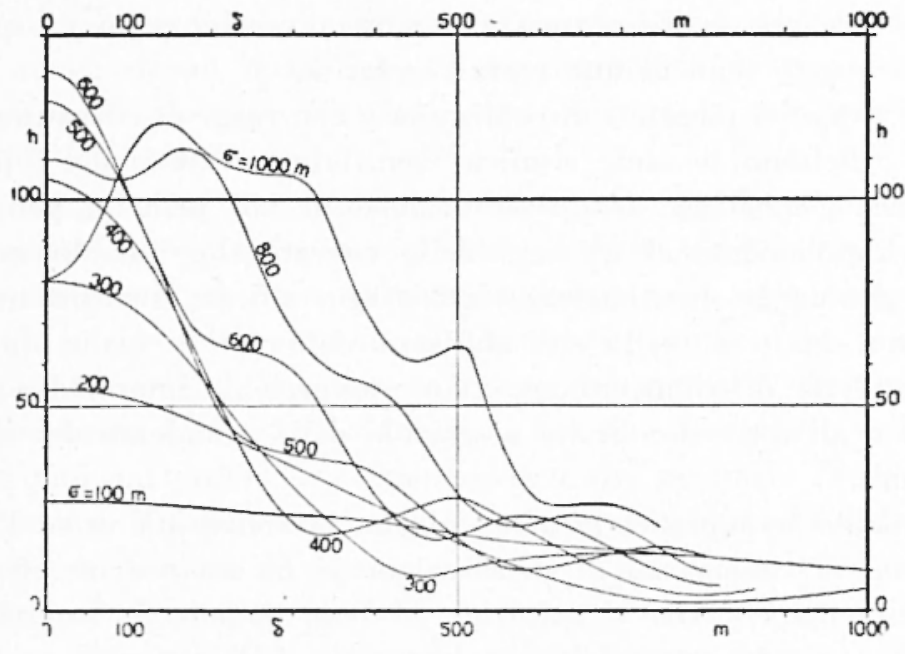

Fig. 5

intemedi fra quelli ottentiti in tale modo e quelli dati, come abbiamo detlo, dalle curve della fig. 2.

Nella figr. 5 riportiano le curve delle ampiezze percentuali relative alla profondità $k$ di $2500 \mathrm{~m}$ e alla lunglıezza d'onda $\lambda$ di $100 \mathrm{~m}$ per le larghezze o delle striscie di 100, 200, 300, 400, 500, 600, 800 e 1000 metri.

Evidentemente tali curve non possono che essere, grosso modo, complementari alle curve della fig. 3 , per grli stessi valori di $\sigma$, ed avere di queste le proprieta che ne permettono l'estensione alle diverse profonditi $h$ delle striscie (considerando lungliezze d'onda tali per cui $\checkmark i k=5001$ e alle diverse lunghezze d'onda $\%$ (moltiplicando i valori di $\delta$ e di $\sigma$ segnali per il valore del rapporto a 100 delle stesse lungliezze d'ondal.

i: facile comprendere come le considerazioni che abbiamo fatto 
nellipotesi dolle superfici riflettenti orizzontali siano valide anche nell'ipotesi che le stesse superfici siano invece inclinate: naturalmente in quest'ultimo caso gli effetti che si rileveranno saranno composti con quelli dovuti alle variazioni delle lunghezze dei percorsi delle onde e alle interferenze delle inclinazioni delle superfici riflellenti, facilmente calcolabili coi semplici principi dell'ottica geometrica.

Le superfici riflettenti interrotte per discordanze si possono considerare agli effetti della riflessione delle onde sismiche come superfici interrotte per faglia, che sopra abbiamo considerato. Ugualmente si può dire per le discontinuità dovute a vecchie ripe di spiaggia, quando queste sono di una certa regolarita.

Le superfici piegate con continuità e con raggi di curvalura molto grandi riflettono le onde sismiche sensibilmente secondo i principi dell'oltica geometrica. L'approssimazione di tali principi però diminuisce rapidamente al crescere della curvatura e quando questa è molto grande la distribuzione dell'energia riflessa aviene in modo del tutto simile a quello che abbiamo visto per le farlie. In realta avremo delle distribuzioni secondo proporzioni intermedie, vicine all'uno o all'altro caso limite a seconda delle grandezze dei ragrari di curvatura.

Quando le superfici piegate volgrono la convessità verso loriorine dei sismi e i sismografi di registrazione si ha sempre un effetto disperdente dell'energia. Ugualmente aviene quando le superfici rolgono la concavità verso l'alto ma hanno raggi di curvature molto piccoli, minori di $\mathbf{k} / 3$ : per valori maggiori degli stessi raggi si ha compre effetto concentrante dell'energia, similmente a quanto abbiamo visto per le lenti.

Possiamo nolare in generale che $\mathrm{i}$ fenomen di diffrazione pur verificandosi per tutte le lunghezze d'onda, risultano più appariscenti per le maggiori lunghezze d'onda. Per le dispersioni dell'energa conseguenti alle discontinuita del mezzo di trasmissione, ossia per l'atlitudine delle onde ad "Aggirare "gli ostacoli, possiamo ritencre chc approssimativamente valga la legge della radice quadrata delle lunghezze d'onda. Vediamo allora che le onde lunghe risultano in roaha più persistenti delle corte, e quindi meglio adatte per i rilievi degrli strati profondi, non solo perché in generale le rocce sono più trasparenti per esse ma anche perché, aggirando più facilmente gli oslacoli, riescono meno contrastate dagli stessi. Questo vantaggio dclle onde lunghe sulle corte in pratica riesce assai diminuito dal Iatto che 
colla magrgiore attitudine ad agrgirare gli ostacoli si hanno delle distorsioni delle superfici d'onda più intense e più estese, tali per cui, in generale, le configurazioni strutturali fittizie che risultano, le quali noi chiameremo con "Strutture simulate», si presentano più appariscenti e più difficili da chiarire nella loro vera natura.

5. Influenzu della diffrazione sulle caralleristiche delle riflessioni. - Albbiamo vislo ai numeri precedenti come per effetto dei fe:momeni della diffrazione si possano avere in pralica delle onde riflesse nelle zone dellombra geometrica con ampiezze tali da dare luogo sui sismogrammi di registrazione a delle riflessioni apparentemente anche ottime, quando le differenziazioni dei terreni sono notevoli lungo le superfici riflettenti. Ora vedremo le caratteristiclue di tempo di queste riflessioni.

Le disposizioni dei sismografi sul tereno di rilievo per rispetto ai pozzetti di scoppio $S$ possono essere di diverse specie: le più usate però sono quelle che noi ahbiamo indicato colle sigle SEA, SES e SWS $\left({ }^{3}\right)$, ossia dello scoppio allineato col profilo dei sismografi (Scoppio Fstremo Allineato), dello scoppio scostato dal profilo in corricpondenza a un estremo (Scoppio Estremo Scostato) e dello scoppio scostato dal profilo in corrispondenza alla mezzaria (Scoppio Medio Scostato). Noi per semplicitá consideriamo soltanto la disposizione SES seconto la quale gli elementi greometrici delle superfici riflettenti vengono calcolati utilizzando i lempi $T_{*}$ relativi alle onde riflesse all'origine dei sisni: le conclusioni cui arriveremo peri saranno facilmente estensibili a tutte le altre disposizioni dei sismograft.

Quando la superficie riflettente or fosse indefunita, piana e inclinata dell'angolo a e il profilo dei sismografi fosse orientato secondo la direzione di massima pendenza, nell'ipotesi che la velocita di trasmissione delle onde sismiche del terreno sia costante ed uguale a $v_{0}$, possiamo determinare gli elementi geometrici, seguendo, come abliamo rletto, il procedimento relativo alla disposizione SES dei sismografi, ponendo

$$
\begin{gathered}
J=\frac{\Sigma}{2 l} \\
\operatorname{sen} \alpha=v_{0} J \quad, \quad l=\tau_{0} \frac{v_{3}}{2} \cos u,
\end{gathered}
$$

ove $\beth$ siano le somme degli incrementi dei tempi delle rifessioni ottenute cogli scoppi coniugati dei profili, $l$ la lunghezza dei profili 
stessi e $\tau_{0}$ il tempo di riflessione allorigine di uno degli estremi cui si vorrliono riferire le riflessioni, evidentemente nell'ipotesi che i pozzetti di scoppio siano posti sui profili dei sismografi; $k$ i la profondita del punto $R_{0}$ di $\pi$ d'intersezione colla normale calata dallo scop-

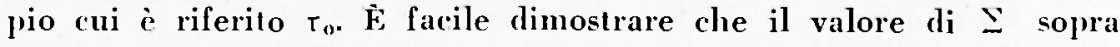
definito $\dot{e}$ uguale alla differenza fra i tempi $\tau_{0}$ relativi agrli scoppi coniugati, ossia relativi ai pozzetti estremi dei profili.

Se invece del piano indefinito $\pi$ abbiamo la striscia piana $\tau_{*}$ di larghezza unitaria considerata nella fig. 4 i tempi $\tau_{0}$ registrati dai sismografi estremi dei profili sono praticamente indipendenti dalla inclinazione $\alpha$ della striscia nel senso normale alla stessa. Se l'inclinazione della striscia nella direzione longitudinale è nulla e supponiamo che i profili siano posti su un piano normale alla striscia grli stessi tempi $\tau_{0}$ risultano funzione soltanto della profondita $k$ della striscia e della posizione dei profili e dei pozzetti di scoppio.

La riflessione delle onde sismiche evidentemente non puo csscre determinata in questo caso applicando le comuni regola dell'ugruaglianza degli angoli dei raggri incidenti e riflessi. Sempre valido invece è il principio della brachistocrona, almeno fino a tanto che si considerano i primi impulsi delle onde registrate. Applicando questo principio otteniamo per la striscia $\pi_{s}$, considerando i pozzetti $S_{\mathrm{i}}$ ed $S_{\mathrm{i}-1}$, naturalmente alla distanza $l$,

$$
1 / 2 \Sigma v_{0}=\sqrt{k^{2}+(\delta+l)^{2}+\left(s-s^{\prime}\right)^{2}}-\sqrt{k^{2}+\delta^{2}+s^{\prime 2}}
$$

ove $s$ è lo scostamento, ossia la distanza del pozzetto $S_{\mathrm{i}}$ dalla linea dei sismografi di regristrazione, e, inoltre, $\delta=k \operatorname{tg} \ell$ ed $s^{\prime}$ data dalla

$$
\frac{s}{s^{\prime}}=1+\sqrt{1+\frac{i^{2}+2 i^{2}}{k^{2}+\delta^{2}}}
$$

Sostituendo il valore di $\varepsilon$ che si ottiene dalla [11] nella [9] e ponendo, al solito, $s=0$ otteniamo, approssimativamente,

$$
J=\frac{1}{v_{0}} \operatorname{sen} \varphi
$$

e quindi $\operatorname{sen} \alpha=\curvearrowright \operatorname{sen} \varphi$.

Vediamo da ciò che, se rilevando una sezione troviamo una striscia riflettente molto stretta disposta ortogonalmente, ammesso che l'energia riflessa sia sufficiente per dar luogo a delle riflessioni attendibili sui sismogrammi, in effetto rileveremo delle pendenze uguali 
ai rapporti fra le distanze orizzontali e le profondità, indipendenti dalla pendenza reale della superficie e quindi avremo delle riflessioni che sensibilmente si ammucchiano tutte in corrispondenza alla striscia.

Dal punto di vista dell'ottica geometrica tale fenomeno può essere assimilato all'effetto disperdente dell'energia sismica di un'anticlinale con piegatura di piccolissimo raggrio di curvatura.

In pratica le striscie molto strette, come si è supposto, solo rarissimamente possono dare luogo a delle riflessioni buone, sia perché le faglie che le generano tolgono la compattezza ai terreni sia perché, in ognni caso, l'energia riflessa non può che essere molto ridotta, come dimostrano le curve delle figure 4 e 5 .

Appena però la larghezza delle striscie diviene sensibile, per esem-

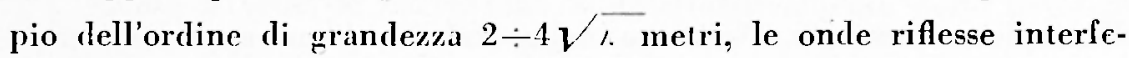
riscono fra di loro e le riflessioni che si registrano risultano di caratteristiche molto più complesse di quelle che abbiamo visto per le striscie sottili.

Avremo prima di tutto che i tempi delle riflessioni relativi ai primi impulsi differiranno da quelli rilevati successivamente in corrispondenza alle diverse fasi dei treni delle onde riflesse.

Utilizzando i primi impeti otterremmo una configurazione delle riflessioni di questo genere: due gruppi di riflessioni ai bordi della striscia con pendenze positive dal centro verso l'estremo crescenti colle distanze dei profili di rilievo, come abbiamo visto che avviene per la striscia sottile, e delle riflessioni coincidenti colla superficie della striscia le quali congiungono i due gruppi. Ma i primi impeti sono di difficilissimo rilievo dai sismogrammi, dato che in generale in questi casi l'energia delle onde riflesse aumenta gradualmente senza nessun incremento iniziale sensibile. Perciò in pratica non si possono che utilizzare i tempi delle fasi successive delle onde riflesse.

Per i sismogrammi rilevati con profili posti in prossimità della normale alla striscia le differenze dei tempi rilevati coi primi impeti saranno sensibilmente uguali a quelle relative ai tempi rilevati colle fasi successive. A mano a mano però che ci si allontanerà dalla normale i valori di tali differenze si scosteranno sempre più. Ciò in relazione al fat to che l'energia riflessa ai sismografi dopo il primo impeto si compone veltorialmente con quella che arriva successivamente dalle parti più lontane della striscia e quindi in ritardo di fase.

Le onde provenienti da una stessa zona non hanno ampiezze costanti: come si sa, il treno di onde che si sviluppa da ogni elemento 
è composto in grencrale di non più di quattro onde complete, con ampiezze crescenti dall'inizio fino a circa metà del treno e decrescenti poi. In pratica quindi avviene che all'incirca per tutta la prima metà del treno le onde in anticipo di fase hanno ampiezze magriori di quelle di fase più rilardata e viceversa per la seconda metà del treno. Questi fatti comporlano l'allungamento dei treni sui sismogrammi e l'aumento graduale degrli incrementi dei tempi (corrispondenti naturalmente alle stesse fasii dall'inizio alla fine del treno, pressoché dai valori corrispondenti agli elementi della striscia più vicini al profilo dei sismografi a quelli corrispondenti agli elementi più lontani.

La costruzione della spirale di Cornu e tutti i calcoli del numero precedente sono stati fatti nell'ipotesi implicita delle onde persistenti, di ampiezza costante. Nel caso della striscia siamo approssimativamente in tali condizioni quando la larghezza della stessa non è molto grande e si considerano le fasi centrali del treno per le quali interferiscono delle onde di ampiezza sensibilmente ugruale per le diverse zone.

Supponendo di operare in tali condizioni vediamo come si possano utilizzare le spirali di Cornu per la determinazione dei valori del rapporto $J$ mediante i tempi delle riflessioni che risultano sui sismogrammi coniugati in presenza di una striscia riflettente $\pi_{s}$ di larghezza $\sigma$, supposto di inclinazione is nulla.

Come abbiamo visto per la determinazione delle ampiezze, dob-

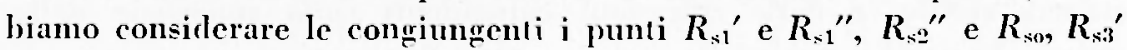
e $R_{\text {ví }}^{\prime}, \ldots \ldots R_{\text {so }}$ e $R_{*:}{ }^{\prime \prime}, R_{* 1}{ }^{\prime \prime}$ e $R_{*}:{ }^{\prime \prime}, \ldots$. e determinare gli angoli che le stesse congiungenti formano colla $R_{s i} R_{s 1}^{\prime \prime}$ che si ha disponendo il profilo dei sismografi in prossimita della verticale per la mezzaria della striscia, cui corrisponde un valore di $J$ nullo delle riflessioni, per $u=0$ come si è supposto. La lunghezza di tali congiungenti deve corrispondere alla larghezza $\sigma$ della striscia riflettente. Se immariniamo che tale larghezza sia piccolissina gli angoli it che si ottengono sono quelli delle tangenti alla spirale colla tangente per il punto

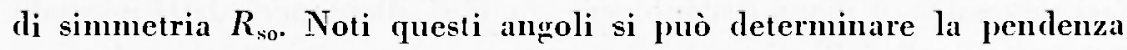
corrispondente a una larghezza qualunque della striscia rifletlente mediando i valori relativi, pressapoco come implicitamente si fa adottando delle congiungenti di lunghezza corrispondente alla larghezza della striscia riflettente.

La determinazione pratica del valore degrli angoli it si può fare in modo semplice riportando, su un sistenna di assi cartesiani orto- 
gonali, come ascisse i valori di $\delta_{1}, \lambda_{2}, s_{.,}, \ldots$. che ahbiamo determinato per la costruzione delle curve della fig. 2, e come ordinate $i$ valori di $\vartheta_{1}$. $\vartheta_{3}, \gamma_{,}, \ldots \ldots$ calcolati mediante la [6]. Sappiamo però che per le stesse profondita $k$ grli angoli $y$ sono inversamente proporzionali alle lunghezze delle onde considerate. Noi perciò nella fig. 6 riporliamo soltanto le curve relative alla lunghezza d'onda $i=$ 100 metri per le solite profondita considerate di 1000,2500 e 5000 metri: da tali curve possiamo ricavare le fasi relative a una qualsiasi lunghezza d'onda molliplicandone i valori per il rapporto $100 \%$, ossia per $4,2,1,1 / 2,7 / 4$ a seconda se si considerano le onde di lunghezza rispettivamente di $2550,100,200,400$ metri.

Come si vede, le curve sono approssimativamente delle parabole

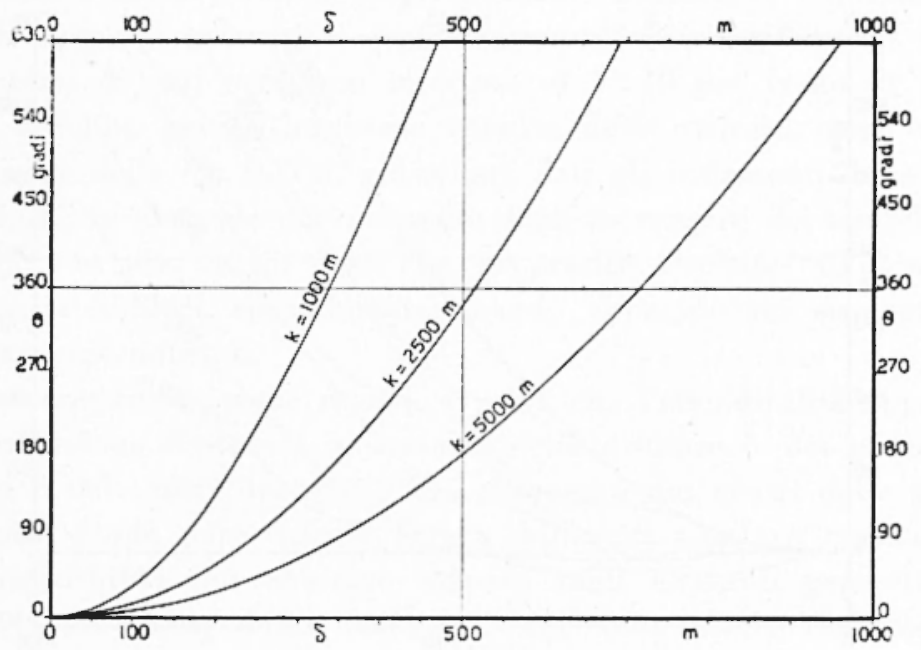

Fig. 6

aventi per asse l'asse delle ordinate. Vediamo anche che $\mathbf{i}$ valori delle fasi per le distanze $\delta$ dalle strisce sono pressoché inversamente proporzionali alle profondità $k$ delle strisce stesse.

Ad uguali valori della fase corrispondono evidentemente delle differenze dei tempi $\Sigma$ che sono direttamente proporzionali alle lunghezze d'onda. Risulta chiaro allora che le stesse differenze dei tempi sono indipendenti dalle lunghezze d'onda considerate.

Note le fasi corrispondenti alle diverse distanze $\delta$ dalla siriscia possiamo determinare le differenze $\Sigma$ dei tempi registrati dai sismografi estremi dei profili di rilievo innalzando dalle distanze $\delta_{1}$ e $\delta_{2}$, corrispondenti alle distanze degli stessi sismografi dalla striscia, le 
rette ordinate rilevando le differenze $\Delta t$ dei valori delle fasi intercettati sulle curve della figura e ponendo

$$
\mathrm{\Sigma}=\frac{\Delta y}{360} \frac{\lambda}{v_{0}} .
$$

Otteniamo allora, per la [9],

$$
J=\frac{1}{2 l} \frac{\left.J_{i}\right)}{360} \frac{\lambda}{v_{0}} .
$$

$\grave{E}$ facile ora vedere che in effetto applicando questa formola coi valori di $\Delta$ i dati dalle curve della fig. 6 otteniamo la [12]. Per esempio, considerando la profondita $k=2500 \mathrm{~m}$ e la lunghezza d'onda

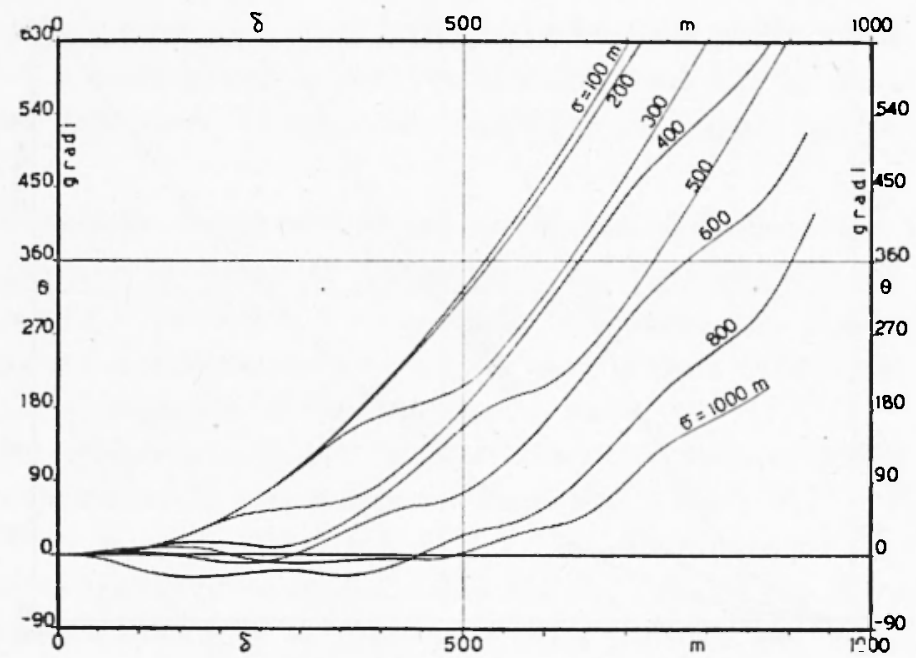

Fig. 7

$\lambda=100 \mathrm{~m}$, otteniamo in corrispondenza alla distanza di $500 \mathrm{~m}$ l'incremento $\delta$ y delle fasi corrispondenti alla lunghezza unitaria $(\delta i)=\Delta i / l$ ) di $\delta \vartheta=1^{\circ}, 40$ e l'angolo $p$ tale che $\operatorname{tog} \varphi=0,20$, per cui $J=\sim 0,20 / v_{\omega}$, valore approssimativamente uguale a quello dato dalla [12], essendo $\operatorname{sen} \varphi=\sim \operatorname{tg} \varphi=0,20$.

Nella fig. 7 riportiamo le curve dei valori delle fasi delle onde riflesse all'origine dei sismi da strisce di larghezza o di 100, 200,300, $400,500,600,800$ e 1000 metri, di inclinazione $u$ nulla, alla profondità $k$ di 2500 metri, per onde di lunghezza $\lambda=100 \mathrm{~m}$.

Per tali curve si possono ripetere le considerazioni che abbiamo fatto a proposito di quelle della fig. 6: mediante esse noi possiamo 
quindi calcolare i valori approssimati delle fasi per valori qualsiasi delle lunghezze d'onda e delle profondita e ampiezze delle striscie riflettenti.

Nella fig. 8 riportiamo in b) le distribuzioni degli elementi geometrici corrispondenti alle riflessioni quali risultano sui normali sismogrammi di rilievo, calcolati coi valori delle fasi dati dalle curve della fig. 7 per la lunghezza dei profili dei sismografi $l$ di $200 \mathrm{~m}$ applicando i normali prontuari hasati sui principî dell'ottica greometrica. Anche in questo caso, naturalmente, si sono supposte le profondita $k$ di $2500 \mathrm{~m}$ e le lunghezze d'onda \%. di $100 \mathrm{~m}$. La velocità $v_{\text {。 }}$ di trasmissione delle onde sismiche del terreno può essere qualunque.

Le tracce delle striscie sono segnate con linee trattegriate qualche millimetro sotto l'inviluppo degli elementi geometrici risultanti.

Gli elementi geometrici segnati sono relativi a riflessioni sui sismogrammi di ampiezza non inferiore al $5 \div 10$ per cento di quella che si arreble per la larghezza infinita delle striscie, come risulta dalle curve della figr. 5. Tali riflessioni, dati gli andamenti delle curve della fig. 7 , in generale non accusano degli incrementi dei tempi molto regolari; ciò non togrlie però che in pratica risultino all'apparenza più che attendibili, specialmente quando appaiono sui sismogrammi con ampiezze notevoli.

Evidentemente, come regrola, averrà che l'attendibilità apparente delle riflessioni diminuiri al crescere delle distanze $\delta$ dei pozzetti di scoppio e dei centri dei profili dei sismografi dai centri delle striscie riflettenti. Onde dare quindi la possibilità di valutare rapidamente tale attendibilità noi abbiamo segnato sugli elementi geometrici le centinaia dei metri delle distanze $\delta$ cui sono relative le riflessioni corrispondenti.

Per comodità, seguendo le abitudini invalse presso i nostri operatori geofisici, chiameremo anche noi con "Riflessioni " gli elementi geometrici che si ricavano col calcolo delle riflessioni vere e proprie che figurano sui sismogrammi.

Vediamo dalla fig. 8 a) che per qualunque larghezza $\sigma$ delle striscie riflettenti si ha sempre, dall'inviluppo delle riflessioni, un andamento anticlinalico.

Gli incrementi degli angoli di fase, come risultano dalle curve della fig. 7 , aumentano in generale al crescere delle distanze $\delta$ dei pozzetti di scoppio e dei profili di rilievo dalle strisce riflettenti: in pratica quindi avverrà che gli andamenti ad anticlinale saranno meglio caratterizzati e le chiusure saranno più appariscenti quando gli 


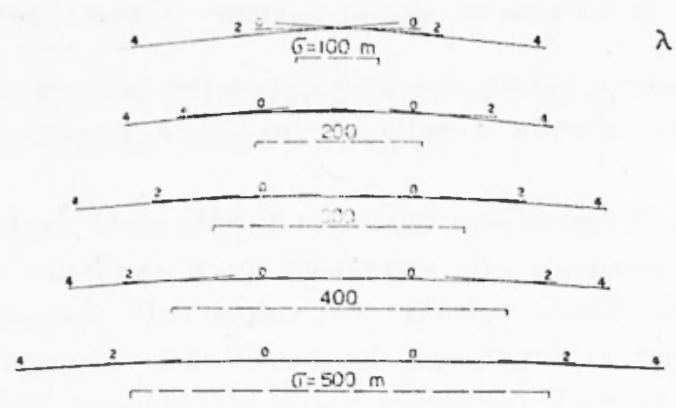

$\lambda=25 \mathrm{~m}$

a)

$$
\begin{aligned}
& 3 \quad 5 \quad 4^{20} \quad 6=100 \mathrm{~m}-0_{24}
\end{aligned}
$$

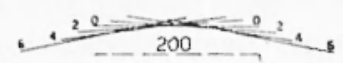$$
\lambda=100 \mathrm{~m}^{*}
$$

b)
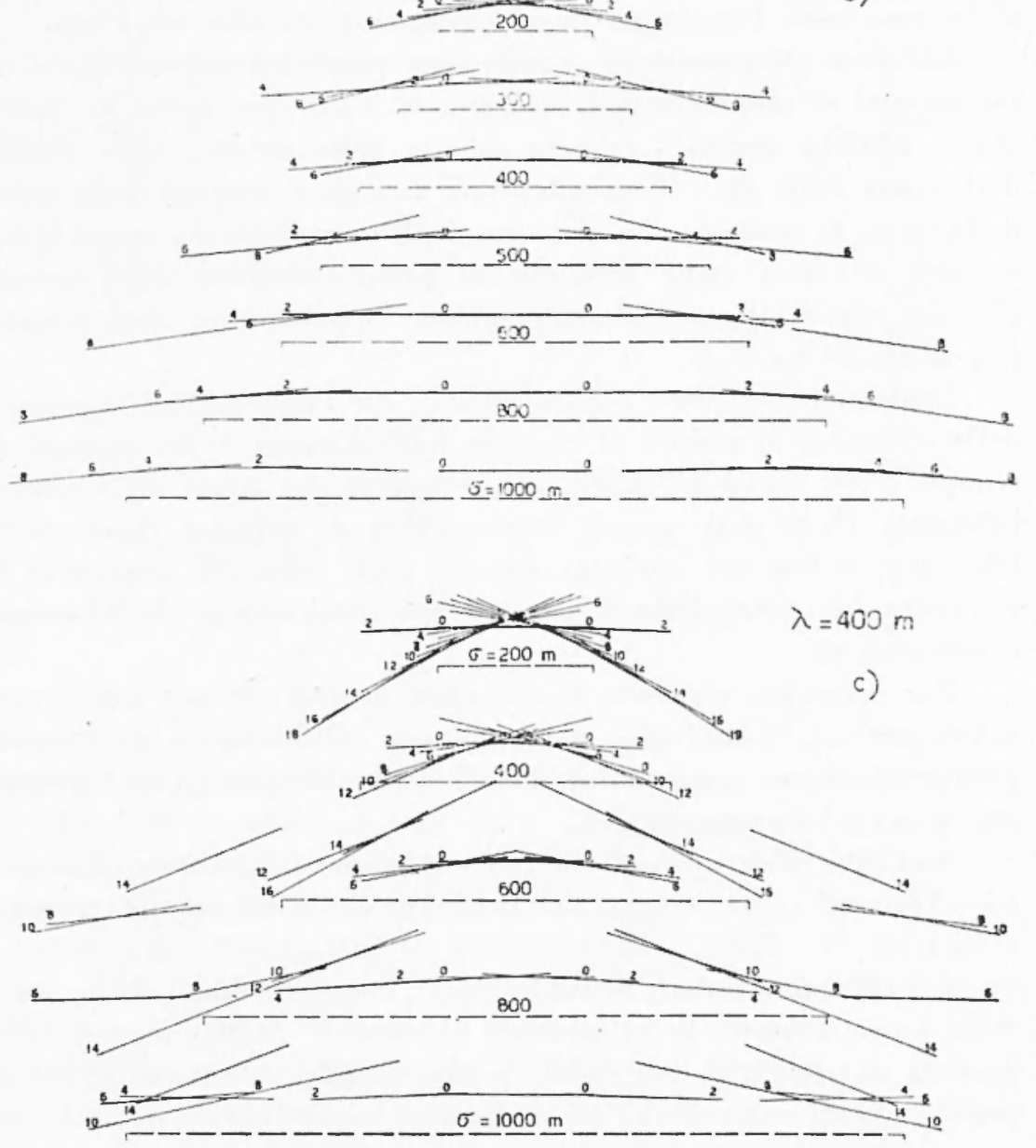

Fig. 8 
strati rifleltenti saranno fortemente differenziati e risulteranno prandi le distanze di rilievo possibile.

Si noti che le irregolarita degli andamenti quali appaiono nella firr. $8 \mathrm{~b}$ in generale risulteranno assai attenuate, se non addirittura eliminate, colle registrazioni delle onde sismiche fatte in trascinamento e colle compensazioni dei valori osservati che gli operatori deb. hono in generale fare per seguire le sequenze delle riflessioni, e che anche per questa ragione le strutture risulteranno in pratica più vistose.

Se adotliamo delle lunghezze $l$ dei profili diverse da quelle di $200 \mathrm{~m}$ considerate otterremo delle distribuzioni delle riflessioni diverse. dalo che $i$ valori delle differenze di fase hi relativi saranno variati. Le differenze delle distribuzioni però saranno sostanzialmente insignificanti per le variazioni che in pratica potranno riuscire utili. tali per cui $l$ sia compresa fra i 100 e i 400 metri.

Pure insignificanti in grenerale risulteranno le variazioni delle distribuzioni conseguenti ai diversi valori che si ottengono cambiando posizione dei pozzetti rispetto alle striscie, per esempio ponendo $i$ pozzetti stessi alle distanze di $100,300,500, \ldots$. metri dalle mezzarie invece che alle distanze di $0,200,400, \ldots$. metri come ablviamo falto per la fig. 8 bi.

Nelle figure 8 cle 8 a riportiamo le configurazioni che invece si ottengrono per le lunghliezze d'onda t. di 400 e di 25 mentri per le larghezze $\sigma$ delle striscie di 200, 400, 600, 800, 1000 metri e di 100, $200,300,400,500$ metri, rispettivamente, sempre per la profondita $h$ di $2500 \mathrm{~m}$ e la lunghezza del profilo di rilievo $l$ di $200 \mathrm{~m}$.

Si può notare che al confronto con le configurazioni relative alla lungheza d'onsla $\lambda=160 \mathrm{~m}$ quelle ottenute per $\lambda=25 \mathrm{~m}$ appaiono molto più regolari ma con chiusure della metà e, viceversa, quelle oltenute per $i=400 \mathrm{~m}$ appaiono in fenerale assai meno regolari e con chiusure pressapoco doppie.

Le riflessioni più esterne segnate corrispondono pure ad ampiezze dell'ordine del $5 \div 10$ per cento di quelle che si avrehbero per larghezze infinite delle strisce.

Variando la profondita $b$ delle strisce e contemporaneamente la lunghezza d'onda $\lambda$ in modo che risulti immutato il prodotto $i k$ otteniamo i medesimi valori delle ampiezze e delle fasi delle figure 5 e 7 . $\Lambda 1$ variare di $\lambda$ però varia anche il valore di $j$, come si vede dalla [15], e con esso varia quello di sence e della pendenza Iga. Questo fatto, 
unito alla variazione della profondita, fa sì che in effetto risultino delle configurazioni simili a quelle delle figure $8 a$ ), b) e $c$ ) ma con dislivelli (chiusure) pressoché ridotte nella stessa proporzione della variazione della lunghezza d'onda. Poiché le ampiezze sono le stesse della fig. 5 le riflessioni risulteranno ugualmente numerose e attendibili come nelle configurazioni delle figure $8 a$ ), $b$ ) e $c$ ).

Nella fig. 9 riportiamo ora le curve dei valori delle fasi $i$ che

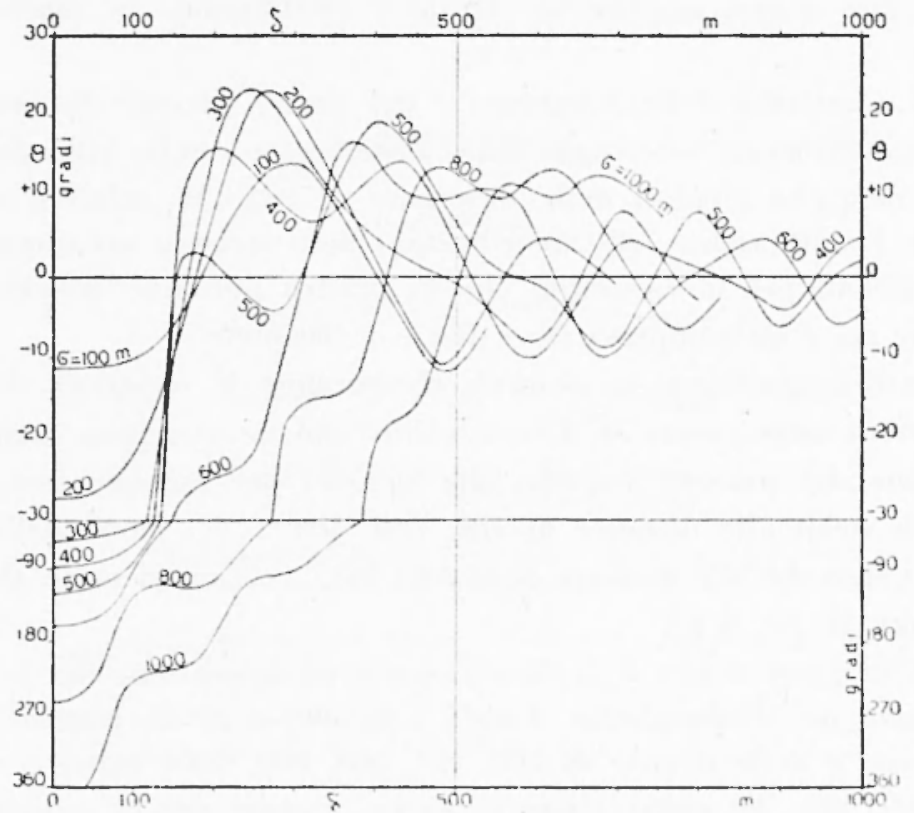

Fig. 9

si hanno per le strisce opache del piano riffettente $\pi$ di larghezze $\sigma$ di $100,200,300,400,500,600,800$ e 1000 metri per la profondita $k$ di $2500 \mathrm{~m}$ di cui abbiamo dato le ampiezze percentuali $h$ nella fig. 3.

Mediante tali curve, date le proprietà che le caratterizzano, al solito possiamo calcolare $\mathrm{i}$ valori approssimati delle fasi relativi a valori qualsiasi delle lunghezze d'onda $i$, delle profondita $k$ e delle ampiezze $\sigma$ delle strisce riflettenti.

Nella fig. 10 a) riportiamo le configurazioni delle riflessioni che, operando nello stesso modo indicato a proposito della fig. 8 , si ottengono dai valori delle fasi della fig. 9, per la solita lunghezza dei profili dei sismografi, $l=200 \mathrm{~m}$, per la profondità $k$ di $2500 \mathrm{~m}$ e per la lunghezza d'onda i di $100 \mathrm{~m}$. 


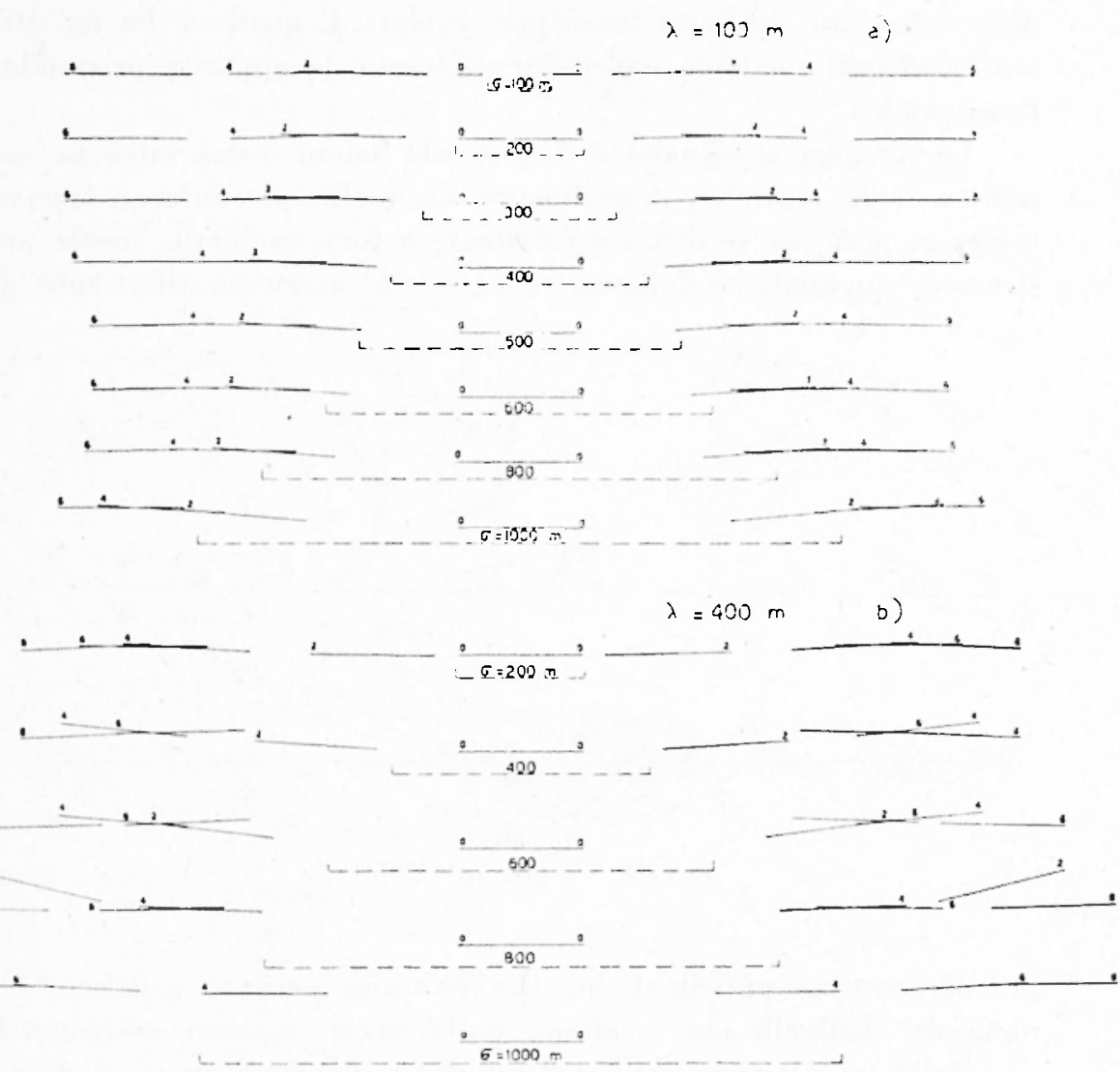

Fig. 10

Vediamo che per tutte le larghezze delle strisce considerate le riflessioni risultano disposte in modo da simulare quasi perfettamente delle strutture sinclinaliche: i dislivelli però sono molto scarsi, al massimo di una trentina di metri per la larghezza di 1000 metri.

Dislivelli maggiori otteniamo per lunghezze d'onda maggiori di $100 \mathrm{~m}$, come si vede dalla fig. $10 \mathrm{~b}$ ). Si vede anche però, da tale figura, che coi dislivelli aumentano le irregolarità delle riflessioni. 'E da notare che per le striscie opache le riflessioni risultano di intensità, e quindi di attendibilità, crescente procedendo dal centro delle strutture similate verso l'esterno, nel senso crescente dei numeri che le contraddistinguono, contrariamente a quanto avveniva nel caso delle strisce riflettenti.

Per le lunghezze d'onda inferiori ai 100 metri le configurazioni 
delle riflessioni risultano ancor più regolari di quelle della fị. 10 a) ma i dislivelli risultano ancor minori, tanto da apparire privi d'interesse pratico.

Le strutture sinclinaliche in generale hamno scarso interesse nella pratica delle ricerche, specialmente di quelle petrolifere; facciamo osservare però che se il piano rifletlente $\pi$ fosse inclinato, invece delle strutlure sinclinaliche delle figure 10 a) e $b$ ) arremmo altrettante cop-

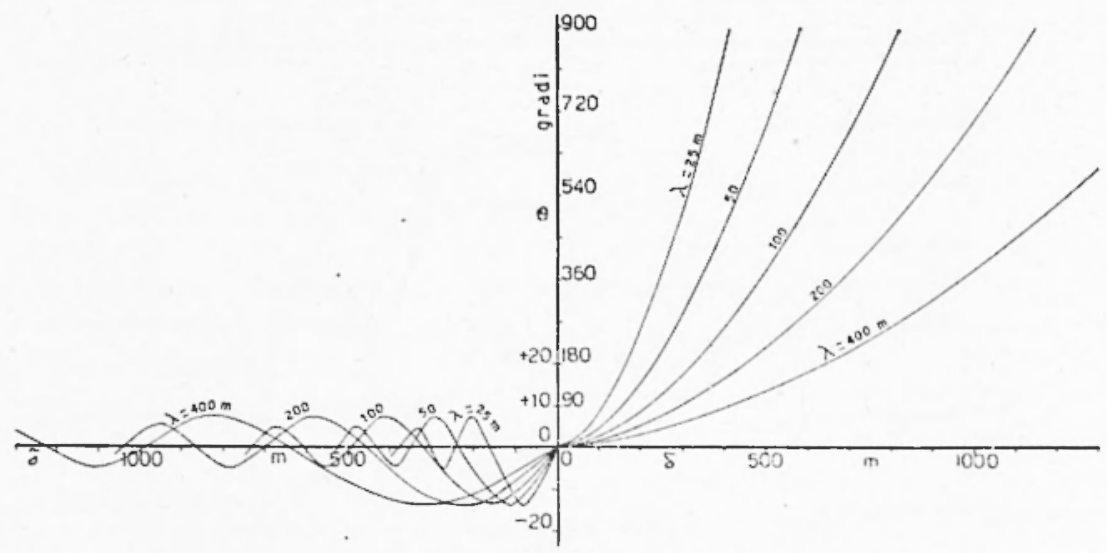

Fig. 11

pie di strutlure anticlinaliche, con chiusure però al massimo della metà dei dislivelli che risultano dalle stesse strutlure sinclinaliche.

Nella fig. 11 riportiamo le curve dei valori degli angoli di fase i) relativi a un semipiano $\pi_{1}$ di profondita $k=2500 \mathrm{~m}$ per le lunghezze d'onda $\lambda$ di $25,50,100,200$ e 400 metri in corrispondenza alle diverse distanze $\delta$ dalla verticale per la retta limite $p_{1}$. Facciamo osservare che le curve a sinistra dell'asse delle ordinate, dalla parte del semipiano riflettente $\pi_{1}$, sono riferite a una scala delle ordinate 9 volte minore di quella cui sono riferite le curve poste alla destra.

Le curve si trasformano l'una nell'altra se si variano le ascisse della radice del rapporto fra le lunghezze d'onda: per esempio dalla $i=25 \mathrm{~m}$ otteniamo quella relativa a $\lambda=109 \mathrm{~m}$ se raddoppiamo $\mathrm{i}$ valori delle ascisse.

I valori delle fasi per le altre profondità si possono otlenere al solito applicando la regola della costanza della radice del prodoto $\lambda k$.

Nelle figure $12 a$ ), bj e $c$ ) riporliamo le configurazioni delle riflessioni che risultano praticamente da un semipiano riflettente $\pi_{1}$ per le lunghezze d'onda $\lambda$ di $25,50,100,200$ e 400 metri; lunghezza dei 


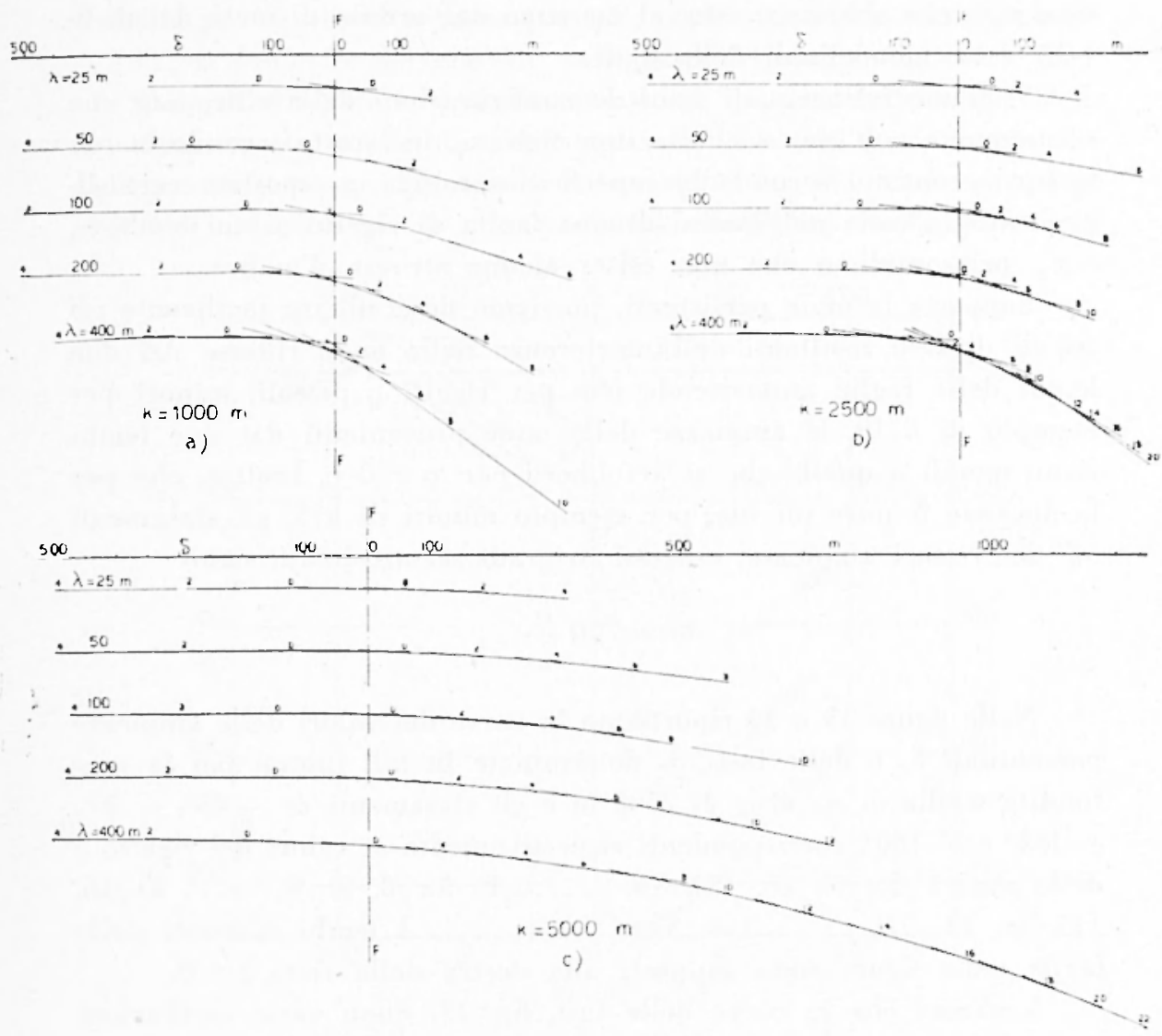

Fig. 12

profili dei sismografi $l$ al solito di $200 \mathrm{~m}$; il semipiano riflettente $T_{1}$ ¿̀ dalla parte delle minori pendenze, alla sinistra del disegno, e la verticale per la retta limite $p_{1}$ (farlia) è individuata dalla $F-F$ a tratto e punto. Le riflessioni più esterne segrnate dalla parte ove manca $\pi_{1}$ corrispondono al solito al limile dell'ampiezza $h$ del $5 \div 10$ per cento.

Ahliamo, come si vede, delle configurazioni monoclinaliche con dislivelli che aumentano al crescere delle lunghezze d'onda $i$ e con curvature che viceversa diminuiscono al crescere della profondita $h$ di $\pi_{1}$.

Se il semipiano $\pi_{1}$ è inclinato verso la partc opposta a quella di $p_{1}$ risultano evidentemente delle configurazioni anticlinaliche, per nulla diverse da quelle "Vere", con chiusure, come pel caso delle 
sinclinali che abliamo visto, al massimo dell'ordine di metà dei dislivelli delle monoclinali della figura.

Vediamo infine quali siano le configurazioni delle riflessioni che si ottengono nell'ipotesi che la superficie $\pi_{1}$, interrotta secondo la retta $p_{1}$, si continui secondo la superficie parallela $\pi$ : spostata verticalmente di $\varrho$, ossia nell'ipotesi di una faglia di rigetto e coi lembi $\pi_{1}$ e $\pi_{0}$ orizzontali, e che non esista alcuna striscia d'ombra.

Supposte le onde persistenti. possiamo determinare facilmente gli angoli di fase risultanti dall'interferenza delle onde riflesse dai due lembi delle faglie ammettendo che per rigetti o piccoli, minori per esempio di $k: 10$, le ampiezze delle onde provenienti dai due lembi siano uguali a quelle che si avrebhero per $\varrho=0 \mathrm{e}$, inoltre, che per le distanze $\delta$ pure piccole, per esempio minori $\mathrm{di} k / 5$, gli sfasamenti fit dei vettori ampiezze, espressi in gradi sessagresimali, siano

$$
\delta_{i}=720 \frac{0}{i} \text {. }
$$

Nelle figure 13 e 14 riportiamo le curve dei valori delle ampiezze percentuali $h_{r}$ e delle fasi $t_{r}$ determinate in tali ipotesi per la profondità media di $\pi_{1}$ e $\pi_{2}$ di 2500 m e gli sfasamenti di $-45^{\circ}$, $-90^{\circ}$, $-135^{\circ}$ e $-180^{\prime \prime}$, corrispondenti rispettivamente ai valori del rigetto $\varrho$ delle serie $\lambda / 16,9 \lambda / 16,17 \lambda / 16, \ldots \ldots \lambda / 8,5 \lambda / 8,9 \lambda / 8, \ldots \ldots 3 \lambda / 16$, $11 \lambda / 16,19 \lambda / 16, \ldots \ldots \lambda / 4,3 \lambda 4 /, 5 \lambda / 4, \ldots$ I lembi ribassati delle faglie nelle figure sono supposti alla destra della retta $\delta=0$.

Vediamo che le curve delle fasi, fig. 13, dopo varie oscillazioni di forma piutlosto complessa e di ampiezze crescenti al crescere degli sfasamenti $\delta_{i}$, tendono alle rette parallele all'asse delle distanze aventi per ordinate $i$ valori degrli sfasamenti corrispondenti, $i_{r}=-45^{\circ}$, $\vartheta_{\mathrm{r}}=-90^{\circ}, i_{\mathrm{r}}=-135^{\circ}$ e $i_{\mathrm{r}}=-180^{\circ}$.

Per lo sfasamento di $-180^{\circ}$ la curva è composta di due parti uguali simmetriche rispetto alla retta $r=0$ spostate nel senso delle ordinate di $-180^{n}$; ugualmente avviene per sfasamenti maggiori. In questi casi mancando orni raccordo delle curve, l'operatore non riscontrerà dai sismogrammi nessun elemento che lo potrà indurre a correlare le riflessioni corrispondenti alle stesse fasi dei treni d'onda le quali appaiono in opposizione o ancor più spostate da una parte e dall'altra delle verticali per le faglie. Lo stesso operatore invece sarà indotto a fare le correlazioni colle onde della seconda falda in anticipo di fase, per cui in effetto grli sfasamenti sono dati dalle simmetriche, rispetto alla retta $\delta=0$, delle curve della fig. 13 : ciò eviden- 
temente per la stessa ragione per cui, cogli spostamenti maggiori di - 180", si fanno le correlazioni colle onde in ritardo di fase.

Per gli altri valori degli sfasamenti $\delta$ it le curve delle ampiezze $h_{r}$ si ottengono da quelle disegnate nella fig. 14 allo stesso modo che ablbiamo indicato per gli angoli di fase della fig. 13. Per le altre lunghezze d'onda $\lambda$ e per le altre profondita delle faglie otteniamo i va-

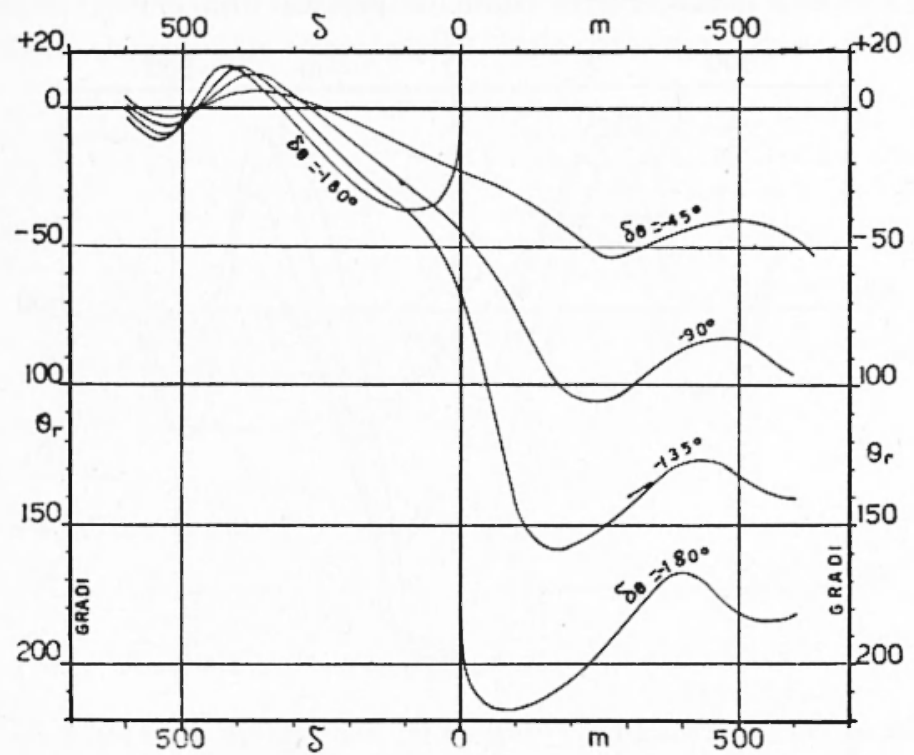

Fig. 13

lori degli angoli di fase e delle ampiezze da quelli delle figure applicando le solite regole che abliamo constatato valide per le altre costruzioni.

Nella fig. 15 riportiamo le configurazioni che praticamente risultano dai rilievi per le lunghezze d'onda $\lambda$ di 25 , 100 e 400 metri per gli sfasamenti di $-45^{\circ}$, $-90^{\circ}$, $-135^{\circ}$ e $-180^{\circ}$. Per quest'ultimo valore si sono fatte le correlazioni in ritardo di fase.

Vediamo che in effetto risultano dei dislivelli perfettamente corrispondenti ai valori degli sfasamenti per le relative lunghezze d'onda. 11 raccordo fra le due falde al solito riesce più regolare e graduale per le maggiori lunghezze d'onda, naturalmente a parità di rigetto.

Le ampiezze, come risultano dalla fig. 14 , sono sempre molto grandi, tali da dare luogo a delle riflessioni sui sismogrammi paragonabili come ampiezze a quelle delle nomali superfici riflettenti con- 
tinue, eccezion falla per il caso $\delta \hat{i}^{\prime}=-180^{\circ} \delta=0$ per cui è nulla. Per gli slasamenti minori di $-180^{\circ}$, dovendosi fare le correlazioni con anticipo di fase, si troveranno in effello dei dislivelli opposti a quelli reali, equivalenti al valore supplementare dello sfasamento, positivo; per esempio, per $\delta^{\prime} t=-270^{\prime \prime}$ si troverà la falda di destra più elevata della sinistra del valore corrispondente allo sfasamento di $+90^{\circ}$. $\dot{E}$ facile comprendere come in pratica una simile eventualiti

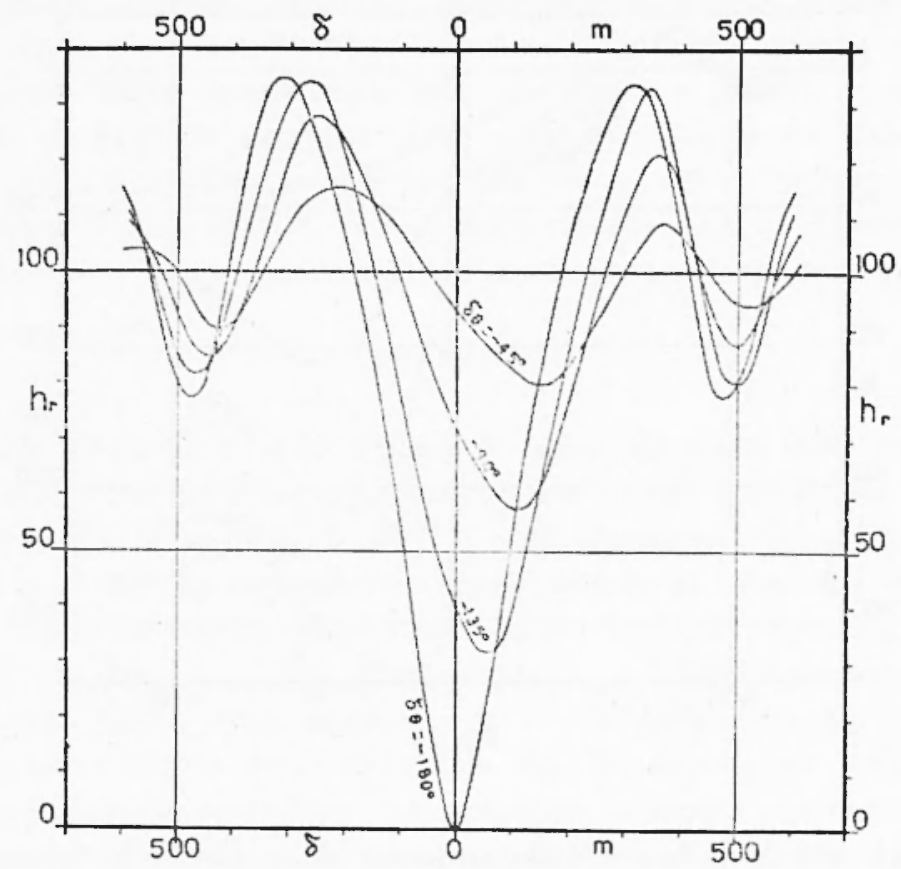

Fig. 11

riesca dannosa: unico elemento di dubbio sulla legitlimità di tali custruzioni invertite si ha dal fatto che risultano correlate, prima e dopo le faglie, delle fasi che nei treni relativi non risullano corrispondenti: per esempio risultano correlate la seconda onda del treno dei sismogrammi rilevati dalla parte del lembo più elevato colla prima onda del treno dei sismogrammi rilevati dalla parte dell'altro lembo.

Per le profondità magriori troviamo delle configurazioni simili ma più estese e più regolari e, viceversa, per le profondità minori delle configurazioni meno sviluppate e meno continue, esatlamente come avviene per le monoclinali simulate della fig. 12 .

Se in corrispondenza alle faglie si avessero delle strisce opache 
risulterebhero evidentemente delle configurazioni simili a quelle della fig. 15: si avrebbero però in ogni caso degli andamenti meno regolari, date le minori ampiezze.

6. Osservazioni. - Le configurazioni delle riflessioni riportate nelle figure potrebbero essere rese più regolari e con minori discontinuità inserendo altri profili fra quelli considerati, limitati al numero strettamente necessario alle correlazioni delle riflessioni delle diverse miFiG. 15

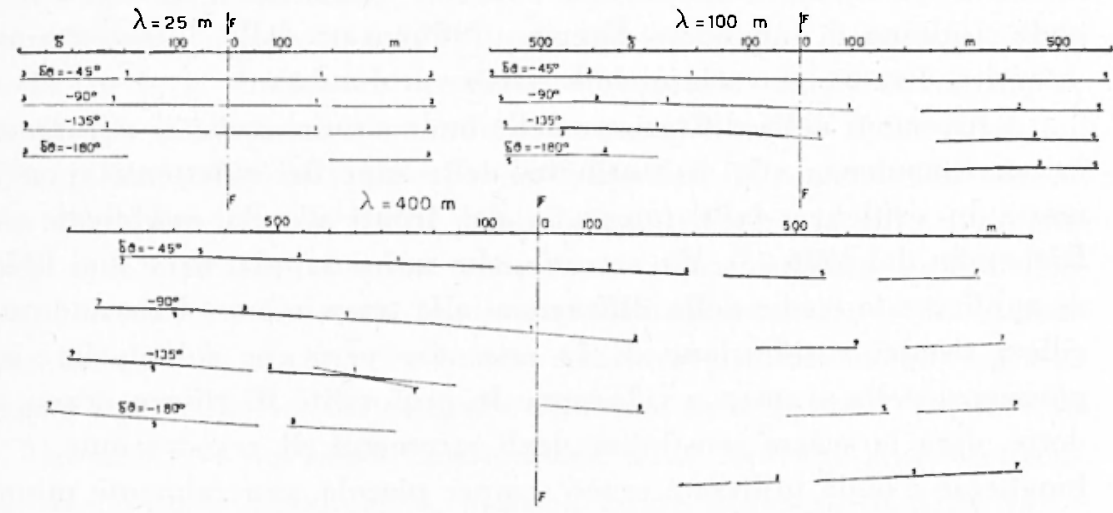

Fig. 15

sure. Già abbiamo detlo però come tali configurazioni in pratica non possano che essere di approssinazione grossolana, specialmente per le riflessioni più esterne, data la scarsa precisione della costruzione grafica delle spirali di Cornu e dato che le lunghezze dei treni d'onda sono limitate e le ampiezze delle onde sono variabili mentre le spirali slesse sono basate sullipotesi delle onde persistenti di ampiezza costante.

Ora osserviamo che colle spirali di Cornu vengono composti gli effetti di

$$
n=(\sqrt{3}-1) \frac{k}{\lambda}
$$

onde complete, per cui le poligronali devono essere arrestate, alla fase di $2 \pi n$, quando ancora sono molto distanti dai centri teorici $C_{1}$ e $C$. i quali venowono in effetto considerati nelle elaborazioni: se per esempio $k=1000 \mathrm{~m}$ e $i=400 \mathrm{~m}$ risulta $n=\sim 1,8$ e la spirale deve essere arrestata alla fase di $\sim 650^{\circ}$ alla quale corrisponde l'ampiezza $\boldsymbol{h}$ di circa l's per cento, secondo la curva $\lambda=400$ della fir. 2 a); con ciò 
evidentemente abbiamo un'ulteriore sensibile riduzione dell'approssimazione, specialmente per gli elementi corrispondenti ai maggiori angoli di fase.

Pel confronto coi fenomeni luminosi ì da tenere presente che mentre per la sismica i rapporti $k / h$ variano da 20 a 200 in oltica tali rapporti sono dell'ordine dei milioni.

Un'ultima causa d'errore abbiamo dal fatto che in generale la velocità di trasmissione delle onde sismiche varia, più o meno gradualmente, colla profondità delle superfici riflettenti c quindi che le onde risultano di lunghezze diverse e influenzate dalla diffrazione con intensità diverse al variare delle stesse profondita.

I fenomeni della diffrazione delle onde sismiche che si sviluppano in corrispondenza alle discontinuiti delle superfici riflettenti vennero messe in evidenza dalle fotografie dei fronti d'onda pulbblicate dal

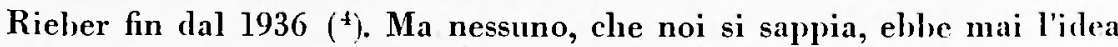
di applicare le teorie della diffrazione alla trasmissione delle onde nei rilievi sismici a riflessione. E da osservare però che colle prime applicazioni della sismica a riflessione le profondita di rilievo erano ridotte, data la scarsa sensibilita degli strumenti di registrazione, e le lunghezze d'onda utilizzate erano sempre piccole, generalmente minori di $50 \mathrm{~m}$, per cui i fenomeni della diffrazione non potevano che ascumere scarsa importanza.

A quei tempi il rilievo delle faglie e, in generale, di tutte le discontinuita delle superfici riflettenti, poteva essere fat to col metodo delle "Correlazioni ", quando gli strati erano bene differenziati e si avevano delle riflessioni caratteristiche sicuramente riconoscibili sui sismogrammi. Coll'adozione del sistema di rilievo a riflessione continuo (") le possibilità delle correlazioni vennero molto estese e con ciò evidentemente anche le possibilità di rilievo delle discontinuità.

Con questi metodi in effetto si rilevano le discontinuità in modo indiretto, correlando le serie di orizzonti rilevate ai due lati delle discontinuità, caratterizzate, queste, da una "Zona d'ombra ", ossia da una zona priva di riflessioni o con riflessioni "Anomale" di andamento sicuramente difforme da quello degli strati della zona: $\grave{i m}$ portante tenere presente però, in proposito, che anche le normali anticlinali possono presentarsi con assenza di riflessioni e provocare dei dislivelli degli strati delle due falde, esattamente come per le faglic.

Attualmente non si hanno altri metodi più efficaci di rilievo delle discontinuità; in generale invece sono aumentate le difficoltà pratiche, 
date le notevolmente maggiori profondita di rilievo che si vogliono raggiungere e le aumentate esigenze nei riguardi della sismica a riflessione.

Le onde lunghe, come ahhiamo visto, sono in generale più persistenti delle corte e sovente danno dei risultati utilizzabili laddove le corte non danno nessuna riflessione attendibile. D'altra parte, quando una zona si rivela sorda coi rilievi normali gli operatori applicano tutti gli accorgimenti utili per avere i risultati e in primo luogo adottano onde il più possibile lunghe e aumentano l'energia degli scoppi (aumentando le cariche e approfondendo i pozzetti). Operando in tale modo, peró, si creano le condizioni favorevoli perché in corrispondenza alle discontinuità, quando esistono, si abbiano delle riflessioni di ampiezze sufficienti anche per le differenziazioni usuali dei terreni. Con ciò si rende generale la possibilità di rilevare le riflessioni anomale e le strutture simulate conseguenti. Praticamente quindi dobbiamo attenderci che tutte le discontinuità diano luogo a delle strutture simulate $e$, anche, che queste strutture risultino meglio caratterizzate e più appariscenti di quelle riportate nelle figure $8,10,12$ e 1.5 .

Le chiusure nel senso longitudinale delle strutture simulate, sempre di carattere anticlinalico, possono essere provocate o dalle variazioni delle inclinazioni " delle superfici riflettenti o dalle variazioni dei dislivelli delle superfici di riflessione delle onde interferenti oppure da entrambe tali cause. $\mathbf{E}$ da tenere presente che rilevando dei profili nel senso longitudinale delle strutture, ossia parallelamente alle linee di discontinuità, si ottengono degli andamenti delle riflessioni perfettamente coerenti con quelli delle riflessioni rilevate coi profili trasversali e che, quindi, si ottengono dei poligoni ipsometrici all'apparenza normali, con errori di chiusura proporzionati alle attendibilità apparenti delle riflessioni.

In mancanza di ogni possibilità di controllare direttamente le caratteristiche delle strutture quali risultano dai rilievi non si può fare altro in pratica che analizzare le riflessioni sui sismogrammi per scorgere in esse le eventuali caratteristiche che abbiamo visto specifiche dell'influenza della diffrazione in corrispondenza alle discontinuità, $e$ in particolare quelle della lunghezza eccessiva dei treni d'onda, della variazione degli incrementi dei tempi colle diverse fasi dei treni $c$, infine, quella delle diverse pendenze e delle diverse configurazioni delle riflessioni che risultano utilizzando lunghezze d'onda notevolmente diverse. 
Data quest'ultima caratteristica converrà in pratica eseguire i rilievi registrando ogni pozzetto con almeno due filıi diversi: uno che sia adalto al migrlior rendimento dell'eneruria riflessa e l'altro il più stretto compatibile colla necessita di avere dei risultati. In tale modo si agevola anche la correlazione delle riflessioni e i rilievi dei tempi delle stesse che proprio nelle zone di difficile rilievo risultano particolarmente lahoriosi $(\%$.

Naturalmente ogni dubbio sull'esistenza delle faglie verrebhe eliminato quando risultassero rilevati i "Liscioni ", ossia le superfici di rottura lungo le quali i lembi delle faglie si sono spostati $\left({ }^{\beta}\right)$. Il caso però non può che essere molto raro in quanto per verificarsi deve avere il concorso di molte condizioni favorevoli, quali quelli di presentare superfici di rottura molto regolari per grandi estensioni, quella che siano posti a contatto terreni con caratteristiche elastiche molto differenziate, ecc.

Milano, Novembre 1952.

\section{RIASSUNTO}

L'iutore riassume $i$ principî di Huyghens, Fresnel e Kirchhoff sulla diffrazione della luce e applica gli stessi principî alla riflessione delle onde sismiche. Dimostra come in corrispondenza alle discontinuità delle superfici riflettenti le onde riflesse alla superficie del suolo non accusino brusche variazioni, come vorrebbero $i$ principt classici dell'ottica geometrica, ma varino gradualmente, sia nelle ampiezze sia negli angoli di fase, e come in conseguenza risultino delle configurazioni per le riflessioni tali da simulare delle strutture continue all'apparenza di grande interesse per la prospezione mineraria. Quindi espone gli accorgimenti che si devono adottare nell'esecuzione dei rilievi e nell'interpretazione geometrica degli stessi per evitare di incorrere in ricostruzioni arbitrarie della tettonica degli strati riflettenti.

\section{SUMMARY}

The author rèsumèes the principles of Huyghens, Fresnel and Kirchhoff on the difraction of light and applies the same principles to the reflection of seismic waves. It is demonstrated how, in cor- 
respondence to the discontinuity of the reflecting surface the uaves reflected to the surface of the soil do uot suffer abrupt variations as predicted by the classical principles of geometric optics, but vary gradually, both in the amplitude and the phase angle, and how, as a consequence, the resulting configurations are such as to simulate continuous structures, and would seem to be of great interest for mineral prospecting. Then he explains the expedients that one must adopt in the execution of the reliefs and in the geometric interpretation of these to acoid incurring arbitrary reconstructions of the tectonic of the reflecting layers.

\section{BIBIIOGR IFIA}

11) Exutco l'Ensico, Otcicr. Francesco Vallardi, Milano, 1932.

- Grongio Valite, Acustica. Francesco Vallardi, Milano, 1928.

- 1). J. W. Cututw, The practical application of acustic principles. E. and F'. N. Spon Ltd, Londral, 1949.

(2) Gustar Kunnump, Vorlesungen äber Mathematische Optik. Lipsia, 1891.

- Maraxo Pitutcci, Fisica classica - Enciclopedia delle matematiche elementari e complemenari. Vol. III. Parte I, Hoepli, Milano, 1917.

(3) Canutuo Coxroxi, L'applicazione del rilievo sismico a riflessione continua, Metano, anno VI, n. l e n. 2, Piddovil, 1952.

(-1) Fhax RieBs:n, Visual presentation of elastic wave patterns under various structural conditions. Geophysics, vol. I, n. 2, 1936.

(ii) Hexir Sacratu, Mapping faults by the reflection method. Geophysics, vol. II, n. 1, 193\%.

(ii) Currus H. Joursox, Locating and detailing faults formations bv means of the geo-sonograph. Geophysies, vol. III, n. 3, 1938. 